\title{
Important Role of SDF-1/CXCR4 Axis in the Homing of Systemically Transplanted Human Amnion- Derived Mesenchymal Stem Cells (Had-Mscs) to Ovaries in Rats with Chemotherapy-Induced Premature Ovarian Insufficiency (POI)
}

\section{Li Ling ( $\nabla$ 304869@hospital.cqmu.edu.cn )} the second affiliated hospital of chongqing medical university https://orcid.org/0000-0001-7712-3277 Jiying Hou

The Second Affiliated Hospital of Chongqing Medical University

\section{Dandan Liu}

The Ninth People's hospital of Chongqing

\section{Dongyuan Tang}

The Second Affiliated Hospital of Chongqing Medical University

\section{Yanqin Zhang}

Wushan County People's Hospital of Chongqing

\section{Qianru Zeng}

The Second Affiliated Hospital of Chongqing Medical University

\section{Heng Pan}

The Second Affiliated Hospital of Chongqing Medical University

\section{Ling Fan}

The Second Affiliated Hospital of Chongqing Medical University

\section{Research}

Keywords: Premature ovarian insufficiency (POI), Human amnion-derived mesenchymal stem cells (hADMSCs), Transplantation, Stem cell home, Stromal cell-derived factor-1 (SDF-1), CXC chemokine receptor 4 (CXCR4)

Posted Date: September 22nd, 2021

DOI: https://doi.org/10.21203/rs.3.rs-915986/v1

License: (9) (i) This work is licensed under a Creative Commons Attribution 4.0 International License. Read Full License 
Version of Record: A version of this preprint was published at Stem Cell Research \& Therapy on February 23rd, 2022. See the published version at https://doi.org/10.1186/s13287-022-02759-6. 


\section{Abstract}

Background: Chemotherapy can induce premature ovarian insufficiency (POI). POI causes multiple sequelae and is currently incurable. Our previous studies have demonstrated that systemically transplanted human amnion-derived mesenchymal stem cells (hAD-MSCs) can home to chemotherapyinduced POI ovaries, and thus reduce ovarian injury and improve ovarian function in rats with POI. However, the cellular mechanisms that direct the migration and homing of hAD-MSCs to chemotherapyinduced POI ovaries are barely understood. This study was to investigate the role of SDF-1/CXCR4 axis in the migration and homing of systemically transplanted hAD-MSCs to chemotherapy-induced POI ovaries and its relevant downstream signaling pathways.

Methods: CXCR4 expression in hAD-MSCs was tested by western blot and immunofluorescence assay. hAD-MSC migration was tested by transwell migration assay. SDF-1 level in rats was detected by ELISA. 72 of female SD rats were randomly divided into the control, POI, hAD-MSCs and hAD-MSCs+AMD3100 groups. POI rat models were established by intraperitoneal injection of cyclophosphamide. For the inhibitor treatment, hAD-MSCs were pretreated with AMD3100 before transplantation. hAD-MSCs labeled with PKH26 were injected into the tail vein of POI rats at $24 \mathrm{~h}$ after chemotherapy. After hAD-MSC transplantation, the homing of hAD-MSCs in ovaries, and ovarian function and pathological changes were examined. To further investigate molecular mechanisms, PI3K/Akt and ERK1/2 signaling pathways were detected.

Results: hAD-MSCs expressed CXCR4. SDF-1 induced hAD-MSC migration in vitro. SDF-1 levels in ovaries and serum significantly increased in $\mathrm{POI}$ rats induced by chemotherapy, and $\mathrm{POI}$ ovaries attracted the homing of hAD-MSCs expressing CXCR4. The block of SDF-1/CXCR4 axis with AMD3100 can significantly reduce the number of hAD-MSCs homing to the POI ovaries, and further reduce their efficacy in POI treatment. The binding of SDF-1 to CXCR4 activated PI3K/Akt signaling pathway, and LY294002 significantly inhibited hAD-MSC migration induced by SDF-1 in vitro. Moreover, inhibition of PI3K/Akt signaling pathway significantly reduced the number of systemically transplanted hAD-MSCs homing to chemotherapy-induced $\mathrm{POI}$ ovaries in rats.

Conclusions: SDF-1/CXCR4 axis partially mediates the migration and homing of systemically transplanted hAD-MSCs to chemotherapy-induced POI ovaries in rats, and PI3K/Akt signaling pathway might be involved in the migration and homing of hAD-MSCs mediated by SDF-1/CXCR4 axis.

\section{Background}

Premature ovarian insufficiency $(\mathrm{POI})$ is a clinical syndrome defined by loss of ovarian activity before the age of 40 years[1]. Untreated POI can cause multiple sequelae, such as infertility, osteoporosis, increased risk of fracture and cardiovascular disease, depression, anxiety, and gynaecological issues[1]. POI can be caused by various factors, mainly including genetic, autoimmune, and iatrogenic factors. About $25 \%$ of patients with $\mathrm{POI}$ are caused by iatrogenic factors, including chemotherapy[4]. Approximately $30 \%$ of 
women before the age of 35 years and $50 \%$ of women between the ages of 35 and 40 years who received chemotherapy were diagnosed with POI[2]. POI is irreversible and currently incurable. Regenerative medicine studies have suggested that mesenchymal stem cell (MSC) transplantation may provide an effective treatment method for POI[3-5]. MSCs have been demonstrated to have significant potential for clinical use. This clinical utility is due to their self-renewal capacity, their convenient isolation, their low immunogenicity permitting allogenic or xenogeneic transplantation, and their potential to differentiate into tissue-specific cell types and promote vascularization[6-8]. Human amnion-derived mesenchymal stem cells (hAD-MSCs) have been proved to not only have the features of MSCs, but also have their unique merits for clinical utility [9-11]. Our previous studies have demonstrated that systemically transplanted hAD-MSCs can partly migrate and home to chemotherapy-induced POI ovaries, and further reduce ovarian injury and improve ovarian function in rats with chemotherapy-induced POI[8]. However, the mechanisms that direct the migration and homing of hAD-MSCs to chemotherapy-induced POI ovaries are poorly understood.

MSC Homing is defined as the arrest of MSCs within the vasculature of a tissue followed by transmigration across the endothelium[12]. Cell migration, an important part of cell homing, is the movement of cells from source to the region where there is a requirement of response or action[13, 14]. Exogenous MSC homing is known as a process where transplanted MSCs are recruited from the peripheral bloodstream to injured or pathological tissue[15]. Efficient homing of stem cells is a prerequisite for successful engraftment of transplanted MSCs. Any improvement of existing cell-based therapeutic approaches will depend on a better understanding of the interaction of stem cells with the environment that leads to homing and tissue integration[16]. Although the molecular mechanisms that direct the migration and homing of MSCs are only partially understood[16], numerous studies have demonstrated that two important detailed mechanisms for the efficiency of MSC homing might be involved[17]. One is that specific ligands or receptors upregulated in injured tissues facilitate trafficking, adhesion, and infiltration of MSCs. The other is that chemokine receptors, selectins and integrins expressed on MSCs are involved in the migration of MSCs across the endothelium and homing to injured tissues.

Chemokines are 8 to $12 \mathrm{kDa}$ peptides that are involved in cell migration and homing[18]. Stromal cellderived factor-1 (SDF-1) is a member of the CXC chemokine subfamily. SDF-1 was considered to be the most potent chemoattractive signal and identified as a stem cell homing factor[19]. SDF-1 is expressed by a wide variety of cells and greatly up-regulated in many tissues induced by"stress," such as injury, inflammation, irradiation or chemotherapy[18-20]. The local elevation of SDF-1 level in the injured tissue is capable of recruiting stem cells to the site of injury where they can support tissue repair and regeneration[19]. Many studies proposed that SDF-1 was one of the most important chemokines and homing factors of stem cells in promoting the migration and homing of stem cells to injured tissues[19, $21,22]$. Studies have found that[23] the chemokine receptor repertoire of human bone marrow-derived mesenchymal stem cells (BM-MSCs) determined their migratory activity, and harnessing the migratory potential of MSCs by modulating their chemokine-chemokine receptor interaction may be a powerful way to enhance homing capacity of stem cells after transplantation. CXC chemokine receptor 4 (CXCR4), a G 
protein-coupled receptor, is the primary chemokine receptor for SDF-1. CXCR4 is widely expressed on various cells, including hematopoietic cells, endothelial and epithelial cells, neuronal cells, progenitor and stem cells[18]. These cells express CXCR4 and migrate along SDF-1 gradients[18]. A number of studies have demonstrated that locally elevated SDF-1, acting via its specific receptor CXCR4 on MSCs, is critical for MSCs homing to injured tissue, including bone marrow, infarcted myocardium, traumatic brain and injured liver[19, 24, 25]. It is widely accepted that SDF-1/CXCR4 axis is associated with the migration and homing of MSCs in vivo[26].

Studies have shown that systemically transplanted MSCs can migrate and home to injured tissues and promote tissue regeneration. SDF- 1 has been found to play a role in the migration and homing of CXCR4positive MSCs to injury tissue and regulate repair activity[27,28]. The migration of MSCs was mediated by upregulated SDF-1 in injury tissue and its specific receptor CXCR4 expressed on MSCs[29, 30]. Mobilization and homing of MSCs were regulated by the interaction between SDF- 1 and CXCR4[19, 24, 25]. There is increasing evidence supporting the central role of the SDF-1/CXCR4 axis in regulating the migration and homing of MSCs to injured tissue. Thus, in this study, we explored whether SDF-1/CXCR4 axis is involved in the migration and homing of systemically transplanted hAD-MSCs to chemotherapyinduced $\mathrm{POI}$ ovaries in rats.

Binding of SDF-1 to CXCR4 can activate multiple signaling pathways to regulate cell migration, proliferation, differentiation, survival and apoptosis[26, 31]. However, the signal networks connecting SDF-1/CXCR4 axis with cell migration and homing are still not well understood. Studies have shown that signaling cascades induced by SDF-1/CXCR4 initiate related pathways in stem cells, including p44/p42 extracellular signal-regulated kinases (ERK1/2) and phosphatidylinositol-3-kinase (PI3K)/Akt, which can affect the chemotaxis and migration of stem cells and result in stem cell mobilization from the bone marrow into the peripheral blood as well as their homing to injury tissue[19, 32]. Studies have shown that CXCR4-mediated chemotaxis is mediated by the activation of PI3K[18]. PI3K activation can result in the phosphorylation and activation of several downstream components such as Akt[18]. It was demonstrated that the PI3K/Akt activation participated in the migration of MSCs, and CXCR4 antagonist, AMD3100, could reverse phospho-Akt expression level and inhibit MSC migration induced by SDF-1/CXCR4 axis[33, 34]. Studies also showed that SDF-1/CXCR4 axis mediated chemotaxis may be driven by the activation of MAPK through ERK1/2[19, 35]. Studies found that SDF-1/CXCR4 axis mediated MSC migration, and the activation of ERK 1/2 downstream signaling pathway by the binding of SDF-1 to CXCR4 was required for MSC migration[35]. Studies demonstrated that SDF-1/CXCR4 axis was critical for MSC migration[36], and SDF-1 pretreatment, acting through its receptor CXCR4 on MSCs, significantly activated PI3K/Akt and ERK1/2 signaling pathways in MSCs, and these effects were partially inhibited by AMD3100[36]. Therefore, in our study, we further explored whether ERK1/2 and PI3K/Akt signaling pathways are involved in the migration and homing of hAD-MSCs mediated by SDF-1/CXCR4 axis.

In this study, the role of SDF-1/CXCR4 axis in the migration and homing of systemically transplanted hAD-MSCs to chemotherapy-induced POI ovaries and its relevant downstream signaling pathways, were 
explored. These findings may provide new evidence for the understanding of the molecular mechanisms involved in the migration and homing of hAD-MSCs to the POI ovaries.

\section{Methods}

The experimental protocols were in compliance with the Helsinki Declaration and approved by the Ethics Committee of the Second Affiliated Hospital of Chongqing Medical University.

\section{Reagents}

Dulbecco's modified eagle medium low-glucose (L-DMEM) and fetal bovine serum (FBS) were purchased from Gibco Co. (Grand Island, NY, USA). Cell Counting Kit-8 (CCK-8), penicillin, streptomycin, TUNEL apoptosis assay kit, Bradford Protein Assay Kit, RIPA lysis buffer and BeyoECL Plus kit were purchased from Beyotime Institute of Biotechnology (Haimen, China). Adipogenic differentiation medium (ADM), osteogenic differentiation medium (ODM), chondrogenic differentiation medium (CDM), Oil Red O, Alician blue and Alizarin Red S were purchased from Cyagen Biosciences Inc. (Suzhou, China). 2-(4amidinophenyl)-6-indolecarbamidine dihydrochloride (DAPI) and phosphate-buffered saline (PBS) were purchased from Boster Biological Technology Co. Ltd (Wuhan, Hubei, China). CXCR4 antibody was purchased from Novus Biologicals (Littleton, CO, USA). Recombinant human SDF-1 was purchased from PeproTech Inc. (Cranbury, NJ, USA). SDF-1, anti-Müllerian hormone (AMH), estradiol (E2) and folliclestimulating hormone (FSH) ELISA kits were purchased from Uscn Life Science Inc. (Wuhan, Hubei, China). Bax, LY294002 and the secondary antibodies were purchased from Cell Signaling Technology Inc. (Boston, MA, USA). DyLight549 was purchased from Abbkine Scientific Co., Ltd (Liyang, Jiangsu, China). AMD3100 was purchased from MedChemExpress (Monmouth Junction, NJ, USA). CleavedCaspase 3, Bcl-2, vascular endothelial growth factor (VEGF) and vascular endothelial growth factor receptor 2 (VEGFR2) were purchased from Affinity Biosciences (Wuhan, Hubei, China).

Cyclophosphamide was purchased from Hengrui Medicine Co., Ltd. (Lianyungang, Jiangsu, China). All other chemicals were purchased from Sigma-Aldrich Co. (St. Louis, MO, USA).

\section{Isolation and culture of hAD-MSCs}

Primary hAD-MSCs were isolated from term amnions according to our previous protocols[9]. Term placentas were collected from healthy donors who received cesarean section at the Second Affiliated Hospital of Chongqing Medical University, Chongqing, China. Written informed consent was obtained from all these donors before collection. hAD-MSCs were cultured in L-DMEM supplemented with $12 \%$ FBS, $100 \mathrm{U} / \mathrm{mL}$ penicillin and $0.1 \mathrm{mg} / \mathrm{mL}$ streptomycin. The third passage of hAD-MSCs were used for the subsequent experiments.

\section{Identification and characterization of hAD-MSCs}


hAD-MSCs were identified according to our previous published protocols[9]. The morphological characteristics and growth of hAD-MSCs were observed and imaged by an inverted microscope (Olympus Corporation, Tokyo, Japan). The expression of MSC surface markers on hAD-MSCs was detected by flow cytometry. To identify the multipotent differentiation of hAD-MSCs, hAD-MSCs were cultured in ADM, ODM and CDM for 21 days, respectively. After staining with Oil Red O, Alizarin Red S or Alician blue, the cells were observed and imaged under an inverted microscope (Olympus Corporation, Tokyo, Japan).

The growth curve of hAD-MSCs was detected by CCK-8 assay according to the manufacturer's instructions. Cells were seeded at a concentration of $5 \times 10^{3}$ cells/well in 96-well plates and cultured for $24 \mathrm{~h}$. Then, the optical density (OD) value at $450 \mathrm{~nm}$ was measured every day for 5 continuous days, using a plate reader (1510model; Thermo Fisher Scientific Oy, Vantaa, Finland).

\section{Transwell migration assay}

A cell migration model in vitro was constructed by transwell chamber (Corning, NY, USA) composed of a membrane filter with $8 \mu \mathrm{m}$ diameter pores suspended in a 6-well plate according to the manufacturer's instructions. hAD-MSCs were placed in serum-free medium at a density of $1 \times 10^{5} / \mathrm{cm}^{2}$ into the upper chamber, and the lower chamber was filled with the same medium containing $2 \%$ FBS. For the inhibitor treatment, hAD-MSCs were preincubated with AMD3100 (44nM, specific inhibitor of CXCR4) or LY294002 (50 $\mu \mathrm{mol} / \mathrm{L}$, specific inhibitor of PI3K/Akt pathway) for $1 \mathrm{~h}$. For the SDF-1 treatment, SDF-1 with the concentration of $0,50,100$ or $150 \mathrm{ng} / \mathrm{mL}$ was added into the lower chamber. After $24 \mathrm{~h}$, migration assays were terminated by retrieving the membrane filter from each group, and hAD-MSCs on the underside of the filter were stained with crystal violet staining solution and counted in 6 visual fields (200x) randomly chosen under microscope (Olympus Corporation, Tokyo, Japan).

\section{Western blot}

For exploring the CXCR4 expression in hAD-MSCs, hAD-MSCs were collected from four different persons. For exploring the mechanisms associated with hAD-MSC migration mediated by SDF-1/CXCR4 axis, hADMSCs were pre-treated with AMD3100 (44nM) or LY294002 (50 $\mu \mathrm{mol} / \mathrm{L})$ for 1 hour followed by the treatment of SDF-1 $(100 \mathrm{ng} / \mathrm{mL})$. After treatment, hAD-MSCs were lysed in RIPA lysis buffer and proteins were isolated after centrifugation. The protein concentrations were determined by the Bradford Protein Assay Kit. The protein samples were separated by SDS-PAGE and subsequently electro-transferred to PVDF membranes (Millipore, USA). After washing, the membranes were blocked with $5 \%$ skim milk for $1 \mathrm{~h}$ and incubated with specific primary antibodies for CXCR4, Akt, phospho-Akt, ERK1/2 or phospho-ERK1/2 overnight at $4^{\circ} \mathrm{C}$. After washing, the membranes were incubated with the secondary antibodies for $1 \mathrm{~h}$ at room temperature, and BeyoECL Plus kit was used for color development according to the manufacturer's instructions. 


\section{Immunofluorescence assay}

For further confirming the expression of CXCR4 in hAD-MSCs, cells were detected by immunofluorescence assay. The cells were fixed, washed and permeabilized in PBS containing $0.5 \%$ Triton X-100 for 30 min. After washing, the cells were blocked with $5 \%$ BSA for $2 \mathrm{~h}$ and incubated with specific primary antibody for CXCR4 overnight at $4^{\circ} \mathrm{C}$. After washing, the cells were incubated with secondary antibodies conjugated with DyLight549 for $1 \mathrm{~h}$ at $37^{\circ} \mathrm{C}$. Then, the cells were counterstained with DAPI and imaged under a laser scanning confocal microscope (Nikon Corporation, Tokyo, Japan).

\section{Labeling and tracking of hAD-MSCs}

To track and locate the transplanted hAD-MSCs in ovarian tissue, the cells were pre-labeled with the PKH26 Red Fluorescent Cell Linker Kits according to our previous published protocols[8] before transplantation. At $24 \mathrm{~h}$ after transplantation of PKH26-labeled hAD-MSCs, ovaries were made into fresh sections and incubated with DAPI, and the sections were imaged under a laser scanning confocal microscope (Nikon Corporation, Tokyo, Japan).

The quantification of homing efficiency of hAD-MSCs within ovarian tissue is typically assessed by averaging the number of PKH26-labeled cells present in 800x microscopic fields randomly chosen from per tissue sample under confocal microscope according to the protocol which has been published[16].

\section{Animal protocols}

Female Sprague-Dawley (SD) rats aged 10 12 weeks were purchased from the Experimental Animal Center of Chongqing Medical University.

To investigate whether SDF-1/CXCR4 axis mediates the homing of hAD-MSCs to chemotherapy-induced $\mathrm{POI}$ ovaries in rats, 72 of female SD rats were randomly divided into 4 groups as follows: control, POI, hAD-MSCs and hAD-MSCs+AMD3100 groups ( $n=18$ in each group). Firstly, the rats were intraperitoneally injected with cyclophosphamide to establish POI models in the POI, hAD-MSCs and hADMSCs+AMD3100 groups according to our previous published protocols[8]. For the inhibitor treatment, hAD-MSCs were incubated with AMD3100 (44nM) for $1 \mathrm{~h}$ before cell transplantation in the hADMSCs+AMD3100 group. Then, at $24 \mathrm{~h}$ after chemotherapy, the rats from the hAD-MSCs and hADMSCs+AMD3100 groups were injected with $0.6 \mathrm{ml}$ of PBS containing $4 \times 10^{6}$ of hAD-MSCs labeled with $\mathrm{PKH}-26$ via the tail vein, while the rats in the control and POI groups were injected with $0.6 \mathrm{ml}$ of PBS. At $24 \mathrm{~h}, 3 \mathrm{w}$ and $6 \mathrm{w}$ after cell transplantation, 6 rats in each group were sacrificed under sodium pentobarbital anesthesia, and samples were collected for the subsequent experiments.

The estrous cycles of rats in each group were recorded by vaginal smears observation as described in our previous published protocols[8]. Regular estrous cycles consist of 4 sequential stages as follows: 
proestrus, estrus, metestrus and diestrus (Fig. 6a). Irregular estrous cycles were also defined as described in our previous published protocols[8].

To further investigate molecular mechanisms involved in the homing of hAD-MSCs mediated by SDF1/CXCR4 axis in vivo, 24 of female SD rats were randomly divided into 4 groups as follows: control, POI, hAD-MSCs and hAD-MSCs+ LY294002 groups ( $n=6$ in each group). The rats were intraperitoneally injected with cyclophosphamide to establish POI models in the POI, hAD-MSCs and hAD-MSCs+ LY294002 groups. For the inhibitor treatment, hAD-MSCs were incubated with LY294002 (50 $\mu \mathrm{mol} / \mathrm{L})$ for $1 \mathrm{~h}$ before cell transplantation in the hAD-MSCs+ LY294002 group. At $24 \mathrm{~h}$ after chemotherapy, the rats from the hAD-MSCs and hAD-MSCs+ LY294002 groups were injected with $0.6 \mathrm{ml}$ of PBS containing $4 \times 10^{6}$ of hAD-MSCs labeled with PKH-26 via the tail vein, while the rats in the control and POI groups were injected with $0.6 \mathrm{ml}$ of PBS. At $24 \mathrm{~h}$ after cell transplantation, rats in each group were sacrificed under sodium pentobarbital anesthesia, and samples were collected for the tracking tests.

\section{Enzyme-linked immunosorbent assay (ELISA)}

To detect the SDF-1 levels in the ovaries and serum of POI rats at $24 \mathrm{~h}$ after chemotherapy, ovarian tissue and serum were collected from the rats in the control and POI groups. Ovarian tissue was homogenized and the supernatant was collected. The SDF-1 levels were detected by ELISA kit according to the manufacturer's instructions.

To detect the serum levels of AMH, FSH and E2 of rats in each group, serum was collected at 0,3 and $6 \mathrm{w}$ after cell transplantation and analyzed using ELISA kits according to the manufacturer's instructions.

\section{Ovarian morphology analysis and follicle counts}

Ten ovaries from each group were collected at $6 \mathrm{w}$ after hAD-MSC transplantation. Ovaries were fixed, dehydrated, paraffin embedded and cut into $5 \mu \mathrm{m}$ sections. The sections were stained with hematoxylin and eosin (HE). The ovarian morphology was observed by an optical microscope (Olympus Corporation, Tokyo, Japan). The follicles in ovaries were classified as primordial, primary, secondary, preovulatory and atretic follicles. The number of follicles was counted as described previously[3, 11].

\section{TUNEL assay}

Ovarian cell apoptosis was tested by the TUNEL apoptosis assay kit according to the manufacturer's instructions. Sections were observed and imaged by an optical microscope (Olympus Corporation, Tokyo, Japan). Nuclei of ovarian apoptotic cells were stained dark brown.

\section{Immunohistochemical staining}


Ovarian tissue sections were incubated with specific primary antibodies for Bcl-2, Bax, cleaved-caspase-3, VEGF and VEGFR2, and then the corresponding secondary antibodies followed by horseradish peroxidase. After that, the sections were stained with 3,3'-diaminobenzidine and counterstained using hematoxylin. Then, the sections were observed and imaged under an optical microscope (Olympus Corporation, Tokyo, Japan). The sections were analyzed as described in our previous published protocols[8]: the number of positive cells graded as 4 (>75\%), $3(51 \sim 75 \%), 2(25 \sim 50 \%), 1(5 \sim 25 \%)$ or 0 $(<5 \%)$, and the staining intensity graded as 3 (brown), 2 (light brown), 1 (light yellow) or 0 (no color). The total score was the sum of the two grades, which was named immunoreactivity score (IS). Ten highpower fields (HPFs, 400x) were randomly chosen from five sections in each group for scoring. The median and range of ISs for each group was calculated.

\section{Statistical analysis}

For all assays, at least three independent experiments were performed. Statistical analysis of data was processed by SPSS 22.0 software (IBM, NY, USA). Data with normal distribution was presented as mean \pm standard deviation, and independent samples $t$-test was used for comparisons between groups. Data with skewed distribution was presented as median and range, and nonparametric Wilcoxon rank test was used for comparisons between groups. Statistical significance was set at $P<0.05$.

\section{Results}

\section{Characterization and identification of hAD-MSCs}

Primary hAD-MSCs were isolated, cultured and identified according to our previous published protocols[9]. The identification of hAD-MSCs was in accordance with the guidelines laid down by International Society of Cell Therapy (ISCT).

The results showed that isolated cells grew as adherent cultures, displayed a fibroblastic morphology and were capable of forming colonies (Fig. 1a-C). The growth curve of hAD-MSCs was investigated by CCK-8 assay (Fig. 1d). The expression of surface markers on hAD-MSCs was similar to BM-MSCs, which has been detected and published in our previous studies[9]. The isolated cells were confirmed to have the ability to differentiate into adipocytes, osteoblasts and chondroblasts under standard differentiating conditions in vitro, which were verified by Oil Red O, Alizarin Red S and Alcian blue staining, respectively (Fig. 1e-g).

These results demonstrate that the isolated cells have the common characteristics of MSCs and are identified as hAD-MSCs.

\section{Expression of CXCR4 in hAD-MSCs}


The expression of CXCR4 in hAD-MSCs was examined by western blot and immunofluorescence assay. The results showed that CXCR4 was stably expressed in hAD-MSCs, and there was no significant difference in different cell clones (Pख0.05; Fig. 2a and b). Red fluorescent signals, which indicated the expression of CXCR4, were observed in hAD-MSCs (Fig. 2C).

\section{SDF-1 induces the migration of hAD-MSCs in vitro}

To explore whether SDF-1 can promote hAD-MSC migration in vitro and the optimal dose of SDF-1 should be chosen for subsequent experiments, transwell migration assay was performed with $0,50,100$ or 150 $\mathrm{ng} / \mathrm{mL}$ of SDF-1. The results showed that compared to the control group ( $0 \mathrm{ng} / \mathrm{mL}$ of SDF-1), the number of hAD-MSCs passing through the membrane induced by SDF-1 was significantly greater in the S1 (50 $\mathrm{ng} / \mathrm{mL}$ of SDF-1), S2 (100 ng/mL of SDF-1) and S3(150 ng/mL of SDF- 1$)$ groups ( $<<0.01$; Fig. 3a and b). Compared to the $S 1$ group, the number of hAD-MSCs passing through the membrane induced by SDF1 was significantly greater in the $S 2$ and $S 3$ groups $(P<0.01$ and $P<0.05 ;$ Fig. $3 a$ and $b)$. However, there was no significant difference between S2 and S3 groups (Pख0.05; Fig. $3 a$ and b). Thus, SDF-1 at the concentration of $100 \mathrm{ng} / \mathrm{ml}$ was selected for the subsequent experiments.

To further explore the role of SDF-1/CXCR4 axis in the migration of hAD-MSCs induced by SDF-1 in vitro, AMD3100, specific inhibitor of CXCR4, was used in the transwell migration assay. The results showed that compared to the control group, the number of hAD-MSCs passing through the membrane induced by SDF-1 was significantly greater in the SDF-1 group ( $P<0.01$; Fig. $3 c$ and $d)$. However, the increased number of hAD-MSCs passing through the membrane induced by SDF-1 was significantly inhibited by AMD3100 ( $P<0.01$; Fig. $3 \mathrm{c}$ and d). Compared to the SDF-1 group, the number of hAD-MSCs passing through the membrane was significantly decreased in the SDF- $1+$ AMD3100 group $(P<0.01$; Fig. $3 \mathrm{C}$ and d).

These results demonstrate that SDF- 1 can induce the migration of hAD-MSCs in vitro, and SDF-1/CXCR4 axis mediates the migration of hAD-MSCs induced by SDF-1.

\section{Elevated levels of SDF-1 in serum and ovarian tissue in POI rats induced by chemotherapy}

To explore whether chemotherapy induces the secretion of SDF-1 in ovaries of rats, the expression levels of SDF-1 in the serum and ovaries in the control and POI groups were measured after chemotherapy. It was found that, compared to the control group, the SDF-1 levels in the serum and ovaries were significantly increased in the $\mathrm{POI}$ group $(\mathrm{P}<0.01$; Fig. $4 a$ and $b)$.

The results demonstrate that chemotherapy can induce the secretion of SDF- 1 in ovaries and cause elevated serum SDF-1 level in rats, and the injured ovaries in rats with chemotherapy-induced POI might attract hAD-MSCs expressing CXCR4. 


\section{SDF-1/CXCR4 axis partially mediates the homing of hAD- MSCs to chemotherapy-induced POI ovaries in rats}

In order to track and locate the transplanted hAD-MSCs in vivo, hAD-MSCs were pre-labeled with PKH26 before transplantation according to our previous published protocols[8], and PKH26-labeled hAD-MSCs showed red fluorescence. The homing and location of transplanted PKH26-labeled hAD-MSCs in ovaries of $\mathrm{POI}$ rats were traced at $24 \mathrm{~h}$ after cell transplantation. The results showed that, $\mathrm{PKH} 26$-labeled cells, which showed red-dotted fluorescent signals, were recruited and located in the interstitium of POI ovaries after transplantation in the hAD-MSCs and hAD-MSCs+AMD3100 groups (Fig. 5a). There were no reddotted fluorescent signals in the control and POI groups as our previous published articles $[8,11]$ (data not shown). A quantitative assessment of the number of hAD-MSCs in ovaries revealed that ovaries in the hAD-MSCs and hAD-MSCs+AMD3100 groups contained $28.60 \pm 21.49$ and $10.00 \pm 6.85$ cells/microscopic field, respectively ( $n=10 ;$ Fig. $5 b$ ). The intensity of red-dotted fluorescent signals and number of hADMSCs in the hAD-MSCs+AMD3100 group were significantly lower than those of hAD-MSCs in the hADMSCs group $(P<0.05$; Fig. 5a and $b)$.

The results demonstrate that ovaries of chemotherapy-induced POI rats are able to attract hAD-MSCs expressing CXCR4 in vivo. SDF-1/CXCR4 axis partially mediates the homing of systemically transplanted hAD-MSCs to chemotherapy-induced POI ovaries.

\section{Effects of hAD-MSCs on ovarian function in rats with chemotherapy-induced POI after the block of SDF-1/CXCR4 axis}

To investigate the effects of hAD-MSC transplantation on ovarian function in rats with chemotherapyinduced POI after the block of SDF-1/CXCR4 axis, estrous cycles and sex hormone levels of rats were analyzed.

From the 3 rd week after hAD-MSC transplantation, $100 \%$ of the rats in the POI group had irregular estrous cycles while $100 \%$ of the rats in the control group had regular estrous cycles (Fig. 6a and b). From the 3rd week after hAD-MSC transplantation, compared to the POI group, the percentages of rats with irregular estrous cycles were lower in the hAD-MSCs and hAD-MSCs+AMD3100 groups (Fig. 6b), while compared to the hAD-MSCs group, the percentages of rats with irregular estrous cycles were higher in the hADMSCs+AMD3100 group (Fig. 6b).

After chemotherapy, compared to the control group, the FSH level was significantly higher $(P<0.05$; Fig. $6 d$ ), while the AMH and E2 levels were significantly lower ( $P<0.05$; Fig. $6 \mathrm{c}$ and e), in the POI, hAD-MSCs and hAD-MSCs+AMD3100 groups, and there was no significant difference between those groups $(P>$ 0.05; Fig. 6c-e). From the 3rd week after hAD-MSC transplantation, compared to the POI group, the FSH level was significantly lower $(P<0.05$; Fig. $6 \mathrm{~d})$, while the $A M H$ and E2 levels were significantly 
higher ( $\mathrm{P}<0.05$; Fig. $6 \mathrm{c}$ and e), in the hAD-MSCs and hAD-MSCs+AMD3100 groups. Moreover, compared to the hAD-MSCs group, the AMH level was significantly lower in the hAD-MSCs+AMD3100 group from the 3rd week after hAD-MSC transplantation ( $P<0.05$; Fig. 6c). Compared to the hAD-MSCs group, the FSH level was significantly higher $(P<0.05$; Fig. $6 \mathrm{~d})$, while the E2 level was significantly lower $(P<0.05$; Fig. $6 \mathrm{e})$ in the hAD-MSCs+AMD3100 group at 6th week after hAD-MSC transplantation.

The results demonstrate that hAD-MSC transplantation can improve ovarian function in rats with chemotherapy-induced POI, and the block of SDF-1/CXCR4 axis with CXCR4 antagonist can reduce its efficacy in POI treatment.

\section{Effects of hAD-MSCs on ovarian injuries induced by chemotherapy in POI rats after the block of SDF-1/CXCR4 axis}

To investigate the effects of hAD-MSC transplantation on ovarian injuries in rats with chemotherapyinduced POI after the block of SDF-1/CXCR4 axis, ovaries were collected for pathological analysis at $6 \mathrm{w}$ after cell transplantation.

The results showed that, compared to the control group, chemotherapy induced follicle loss, vascular damage, and tissue fibrosis in ovaries in the POI group (Fig. 7a), while compared to the POI group, hADMSC transplantation reduced follicle loss, vascular damage, and tissue fibrosis in the hAD-MSCs and hAD-MSCs+AMD3100 groups (Fig. 7a). Compared to the control group, the number of primordial follicles and growth follicles, including primary, secondary, and preovulatory follicles, was significantly lower in the $\mathrm{POI}$ group ( $\mathrm{P}<0.05$; Fig. $7 \mathrm{a}$ and $\mathrm{b}$ ), while compared to the $\mathrm{POI}$ group, the number of primordial and primary follicles was significantly greater in the hAD-MSCs and hAD-MSCs+AMD3100 groups $(P<0.05$; Fig. 7a and b). Moreover, compared to the hAD-MSCs group, the number of primordial was significantly lower in the hAD-MSCs+AMD3100 group $(P<0.05$; Fig. 7a and $b)$.

Compared to the control group, the number of apoptotic granulosa cells (GCs) in ovaries was significantly greater in the POI group (Fig. 7c). Compared to the POI group, the number of apoptotic GCs in ovaries was significantly lower in the hAD-MSCs and hAD-MSCs+AMD3100 groups (Fig. 7c). Moreover, compared to the hAD-MSCs group, the number of apoptotic GCs in ovaries was significantly higher in the hADMSCs+AMD3100 group (Fig. 7c).

Compared to the control group, the expression levels of Bax and cleaved-caspase-3 (proapoptotic protein) were significantly higher $(\mathrm{P}<0.01$; Fig. $7 \mathrm{~d}-\mathrm{i})$, while the expression levels of $\mathrm{Bcl}-2$ (antiapoptotic protein), VEGF and VEGFR2 were significantly lower $(\mathrm{P}<0.01$; Fig. 7d-i), in the POI group. Compared to the POI group, the expression levels of Bax and cleaved-caspase-3 were significantly lower $(P<0.01$; Fig. $7 d-i)$, while the expression levels of Bcl-2, VEGF and VEGFR2 were significantly higher $(P<0.01$; Fig. $7 d-i)$, in the hAD-MSCs and hAD-MSCs+AMD3100 groups. Moreover, compared to the hAD-MSCs group, the 
expression levels of Bax and cleaved-caspase-3 were significantly higher in the hAD-MSCs+AMD3100 group $(P<0.01$; Fig. $7 d-i)$.

These results demonstrate that hAD-MSC transplantation can reduce ovarian injuries, inhibit GC apoptosis and proapoptotic protein expression, and promote antiapoptotic protein, VEGF and VEGFR2 expression in the ovaries of POI rats, and the block of SDF-1/CXCR4 axis with CXCR4 antagonist can reduce its efficacy.

\section{Activation of PI3K/Akt signaling pathway by SDF-1 in hAD- MSCs}

To explore the mechanisms that transmit SDF-1/CXCR4 axis mediated chemotactic homing signals within hAD-MSCs, PI3K/Akt and ERK1/2 signaling pathways were detected.

Following the treatment of hAD-MSCs with SDF-1, western blot was performed to test the key proteins of $\mathrm{PI} 3 \mathrm{~K} / \mathrm{Akt}$ and ERK1/2 signaling pathways. It was found that significant phosphorylation of Akt was induced by SDF-1 $(P<0.01$; Fig. 8a and $c)$. However, the phosphorylation level of ERK1/2 was not increased significantly after the SDF- 1 treatment $(P>0.05 ; 8 a$ and $b)$. The expression level of phosphoAkt increased significantly after the SDF-1 treatment, while AMD3100 or LY294002 pretreatment significantly inhibited the elevated expression level of phospho-Akt induced by SDF-1 $(P<0.01$; Fig. $8 d$ and e).

The results demonstrate that the binding of SDF-1 to CXCR4 can lead to phosphorylation of Akt and activate PI3K/Akt signaling pathway, and SDF-1/CXCR4 and PI3K/Akt signal pathways can be synchronously activated in hAD-MSCs by SDF-1 in vitro. PI3K/Akt signaling pathway might be downstream chemotactic homing signals in hAD-MSCs that could be activated by SDF-1/CXCR4 axis.

\section{PI3K/Akt signaling pathway is involved in the migration of hAD-MSCs mediated by SDF-1/CXCR4 axis}

To explore the effects of PI3K/Akt signaling pathway on the migration of hAD-MSCs mediated by SDF1/CXCR4 axis, cells were pretreated with inhibitor LY294002 before SDF-1 treatment.

The results showed that compared to the control group, the number of hAD-MSCs passing through the membrane induced by SDF-1 was significantly greater in the SDF-1 group $(P<0.01$; Fig. 9a and $b)$. Compared to the SDF-1 group, the number of hAD-MSCs passing through the membrane induced by SDF1 was significantly reduced by LY294002 pretreatment in the SDF- $1+L Y 294002$ group $(P<0.01$; Fig. 9a and b). 
These results demonstrate that PI3K/Akt signaling pathway is involved in the migration of hAD-MSCs mediated by SDF-1/CXCR4 axis.

\section{Inhibition of PI3K/Akt signaling pathway reduces hAD-MSC homing in vivo}

To further confirm whether PI3K/Akt signaling pathway is involved in the homing of systemically transplanted hAD-MSCs to chemotherapy-induced POI ovaries in rats, cells were pretreated with inhibitor LY294002 before transplantation.

The homing and location of transplanted PKH26-labeled hAD-MSCs in ovaries of POI rats were traced at $24 \mathrm{~h}$ after cell transplantation. The results showed that, $\mathrm{PKH} 26$-labeled cells with red-dotted fluorescent signals were recruited and located in the interstitium of POI ovaries after transplantation in the hAD-MSCs and hAD-MSCs+ LY294002 groups (Fig. 10a). There were no red-dotted fluorescent signals in the control and POI groups as our previous published articles[8, 11] (data not shown). A quantitative assessment of the number of hAD-MSCs in ovaries revealed that ovaries in the hAD-MSCs and hAD-MSCs+LY294002 groups contained $20.00 \pm 17.22$ and $8.70 \pm 6.08$ cells/microscopic field, respectively ( $n=10 ;$ Fig. $10 \mathrm{~b})$. The intensity of red-dotted fluorescent signals and number of hAD-MSCs in the hAD-MSCs+ LY294002 group were significantly lower than those of hAD-MSCs in the hAD-MSCs group $(P<0.05$; Fig. 10a and $b)$.

The results demonstrate that inhibition of PI3K/Akt signaling pathway can reduce systemically transplanted hAD-MSCs homing to chemotherapy-induced POI ovaries, and PI3K/Akt signaling pathway might be involved in the homing of hAD-MSCs mediated by SDF-1/CXCR4 axis in vivo.

\section{Discussion}

This study shows that SDF-1/CXCR4 axis partially mediates the migration and homing of systemically transplanted hAD-MSCs to chemotherapy-induced POI ovaries in rats, and PI3K/Akt signaling pathway might be involved in the migration and homing of hAD-MSCs mediated by SDF-1/CXCR4 axis.

$\mathrm{POI}$ can be caused by various factors, including chemotherapy, which results in multiple sequelae and has consequences for health in women. POI is irreversible and currently incurable. Regenerative medicine studies have suggested that MSC transplantation may provide an effective treatment method for POI[35]. Our previous studies have also demonstrated that systemically intravenously transplanted hAD-MSCs can partly home to chemotherapy-induced POI ovaries, and reduce ovarian injury and improve ovarian function in rats with POI[8,37]. Migration and homing of MSCs to injured tissue are essential for them to fulfill their functions in tissue regulation and repair. It has been reported that the ovary is the primary target organ injured by the toxic effects of cyclophosphamide in clinical chemotherapy[38], which can induce follicular loss, vascular damage, GC apoptosis, and tissue fibrosis in ovaries and cause POI[39, 40]. Thus, cyclophosphamide was chosen to establish POI rat models in our study. Interestingly, we found that, after hAD-MSC transplantation, no or few PKH26-labeled hAD-MSCs located in livers, hearts, brains, 
kidneys, and lungs, and most PKH26-labeled hAD-MSCs homed to ovaries in chemotherapy-induced POI rat models[8], which indicate tissue-specific homing of injected hAD-MSCs to injured tissue in vivo. However, the cellular mechanisms that direct the migration and homing of hAD-MSCs to chemotherapyinduced POI ovaries are barely understood.

Chemokines have been found to act as strong chemoattractants for the homing of stem cells[24]. Some studies have found that chemokines, released from tissue or endothelial cells, and chemokine receptors, expressed on the MSCs, may partly mediate MSC actively homing to specific sites and subsequent retention in tissue[16]. Chemokines upregulated in injure tissue and chemokine receptors expressed on MSCs might be involved in the efficiency of MSC homing[17, 27, 41].

SDF-1 is well known as a key regulator of migration and homing of CXCR4-positive stem cells to injury tissue[41, 42]. The SDF-1 concentration gradient is a driving force for stem cell migration[41]. Studies showed that the expression of SDF-1 was present at extremely low levels or even not originally identified in normal ovaries[43, 44]. However, after injury, the chemokine SDF-1 level increased in tissue, which is critical for the chemotaxis of stem cells homing to the injured or ischemic tissue[24, 27, 45-49]. It was found that SDF-1 was the key cytokine in regulating local inflammation and tissue repairment by recruiting bone marrow stem cells in the case of organ and tissue damage[50,51]. Previous researches showed that SDF-1 expression up-regulation after myocardial infarction mainly enhanced retention of transplanted MSCs in the injured myocardium[52, 53]. Kitaori et al. found that the SDF-1 level was elevated in the fracture site, which recruited MSCs homing to the site of injured bone and promoted bone repair[54]. It was reported that the expression level of SDF-1 increased in the injured ovaries caused by chemotherapy[55]. In our studies, we also found that, the SDF-1 levels in the serum and ovarian tissue of normal rats were very low in the control group, while the SDF-1 levels in the serum and ovarian tissue of $\mathrm{POI}$ rats induced by chemotherapy were significantly increased in the POI group. Thus, we speculated that the upregulated SDF-1 levels in serum and ovaries of POI rats might regulate the homing of transplanted hAD-MSCs from the systemic circulation to ovaries, and further experiments were done for this speculation.

CXCR4, a specific chemotactic receptor for SDF-1, which is found to express in numerous types of adult stem cells[24], is a critical regulator of stem cell mobilization and recruitment. SDF-1 can mobilize stem cells into injured tissues by binding CXCR4 receptor on stem cells[56, 57]. Although molecular mechanisms that direct the migration and homing of MSCs are only partially understood[16], CXCR4 is believed to play an important role in this process[35]. Studies reported that MSCs strongly expressed functionally active CXCR4[23, 24]. However, studies also showed that CXCR4 expression might vary in MSCs from different animal species and/or tissue types[58], and MSCs might lose the expression of homing molecules, such as CXCR4, during expansion in vitro[59, 60]. In our studies, the third passage of hAD-MSCs were used for the experiments, and hAD-MSC population from different clones was proved to stably express CXCR4 protein. The results confirm the abundant CXCR4 expression in hAD-MSCs, which is similar to CXCR4 expression in other types of $\operatorname{MSCs}[24,35,61]$. 
As described above, SDF-1 is critical for the organ-specific chemotaxis and homing of stem cells to injured tissue[62], and these effects are mediated by SDF-1 binding to its receptor CXCR4 located on the surface of stem cells[63]. In our studies, we found that the SDF-1 levels in the ovarian tissues and serum were significantly increased in rats with chemotherapy-induced POI, and hAD-MSCs expressed CXCR4. SDF-1 is a ligand and strong chemokine for CXCR4 expressing cells[35]. Thus, we further speculated that SDF-1/CXCR4 axis might play a role in the process of the migration and homing of hAD-MSCs to chemotherapy-induced POI ovaries.

Studies showed that the homing of stem cells was associated with SDF-1 release from injured tissue as a chemoattractant and SDF-1/CXCR4 axis played an important role in the homing of stem cells[60, 64, 65]. SDF-1/CXCR4 axis interactions are implicated as a critical axis regulating stem cell trafficking and homing to the injured tissue[66]. SDF-1/CXCR4 axis has been proved to direct the migration and homing of stem cells related to injury repair in many species and tissue types[24, 67]. SDF-1/CXCR4 axis is also vital for modulating MSC migration and homing[35,60]. Some studies found that SDF-1/CXCR4 axis was a prerequisite for the homing of MSCs, SDF- 1 was a chemoattractive signal to regulate CXCR4 ${ }^{+} \mathrm{MSC}$ homing to injured tissue[ $41,60,68,69]$. In our studies, we detected a strong chemoattractive activity of SDF-1 to hAD-MSCs. We found that the elevated SDF-1 level promoted the migration of hAD-MSCs in vitro, and the increased number of migrated hAD-MSCs induced by SDF-1 was significantly reduced by CXCR4 specific antagonist, AMD3100. These results demonstrate that SDF-1 can induce migration of hAD-MSCs in vitro, and SDF-1/CXCR4 axis mediates the migration of hAD-MSCs induced by SDF-1. To further explore whether SDF-1/CXCR4 axis is involved in the migration and homing of hAD-MSCs to chemotherapy-induced POI ovaries in vivo, hAD-MSCs were pretreated with AMD3100 and systemically intravenously transplanted into POI rats. We found that, compared to the hAD-MSCs group, the number of hAD-MSCs homing to POI ovaries significantly decreased in the hAD-MSCs + AMD3100 group. These results demonstrate that SDF-1/CXCR4 axis is involved in the migration and homing of hAD-MSCs to chemotherapy-induced POI ovaries in rats. However, we also found that AMD3100 could only partly reduce the number of hAD-MSCs homing to POI ovaries, which implies that SDF-1/CXCR4 axis is not the only pathway involved in the migration and homing of hAD-MSCs in vivo. Thus, SDF-1/CXCR4 axis partially mediates the migration and homing of systemically transplanted hAD-MSCs to chemotherapyinduced POI ovaries in rats.

SDF-1/CXCR4 pathway is known as an upstream switch for many migration pathways. The binding of SDF-1 to CXCR4 can activate multiple downstream signaling pathways in target cells, resulting in phosphorylation of downstream effectors such as Akt, ERK1/2, focal adhesion kinase (FAK) and p38 [18], which in turn regulates various biological effects, including cell motility, chemotaxis and adhesion[18, 70, 71]. PI3K/Akt signal pathway, which is a downstream pathway of SDF-1/CXCR4, has been found to regulate cell migration[26, 72]. Activation of PI3 kinase (PI3k) by SDF-1 in the SDF-1/CXCR4 axis mediated chemotaxis is essential for cell migration, which in turn results in the phosphorylation of downstream molecules such as Akt [34, 35]. It has been reported that PI3K/Akt is an important downstream pathway that could regulate the migration of various stem and progenitor cells, including 
bone marrow MSCs, via the SDF-1/CXCR4 axis[19, 26, 34]. Studies also showed that SDF-1/CXCR4 axis mediated chemotaxis may be driven by the activation of MAPK through ERK1/2[19,35]. Chen et al. found that SDF-1-mediated migration of cardiac stem cell can be inhibited by the block of CXCR4, and the inhibition involved the decrease of phospho-ERK1/2[73]. Li et al found that SDF-1/CXCR4 axis mediated MSC migration, and the activation of ERK $1 / 2$ downstream signaling pathway by the binding of SDF- 1 to CXCR4 was required for MSC migration[35]. Thus, to further investigate the molecular mechanisms involved in the migration and homing of hAD-MSCs to chemotherapy-induced POI ovaries mediated by SDF-1/CXCR4 axis, PI3K/Akt and ERK1/2 signaling pathways were explored in this study.

We found that the binding of SDF-1 to CXCR4 can activate PI3K/Akt signaling pathway, and SDF1/CXCR4 and PI3K/Akt signal pathways were synchronously activated in hAD-MSCs by SDF-1. AMD3100 inhibited the SDF-1/CXCR4 axis and further indirectly inhibited the activation of PI3K/Akt signal pathway, which can significantly reduce the migration of hAD-MSCs induced by SDF-1 in vitro, while LY294002 directly inhibited the activation of PI3K/Akt signal pathway, which can also significantly reduce the migration of hAD-MSCs induced by SDF-1 in vitro. To further confirm the effects of PI3K/Akt signaling pathway on the homing of systemically transplanted hAD-MSCs to chemotherapy-induced POI ovaries in vivo, cells were pretreated with inhibitor LY294002 before transplantation. We found that compared to the hAD-MSCs group, LY294002 intervention significantly reduced the number of transplanted hAD-MSCs homing to POI ovaries in the hAD-MSCs + LY294002 group. Therefore, it is concluded that PI3K/Akt signaling pathway plays an important role in facilitating the migration and homing of hAD-MSCs to chemotherapy-induced POI ovaries, which is in tandem with SDF-1/CXCR4 axis and might be the downstream pathway during hAD-MSC migration and homing to the injured POI ovaries. PI3K/Akt signaling pathway might be involved in the migration and homing of hAD-MSCs mediated by SDF1/CXCR4 axis.

On the other hand, our studies also showed that the binding of SDF-1 to CXCR4 was not able to activate ERK1/2 signaling pathway in hAD-MSCs, which is not consistent with some other studies[35, 73]. We consider that the activation of downstream signaling pathways by SDF-1/CXCR4 axis related to cell migration might vary in stem cells from different animal species, tissue types and/or cell types. However, much remains to be done to further define these signaling pathways.

At present, local transplantation of MSCs is usually invasive, and the potential for minimally invasive delivery of MSCs via systemic infusion is still of particular interest. Systemic intravenous transplantation of MSCs for the treatment of POI has been shown to be efficacious and safe[3-5]. However, the low rate of cell homing, retention and survival after cell transplantation is a limitation of cell-based therapy for POI in current studies, and increasing homing rate of systemically transplanted MSCs to POI ovaries may improve therapeutic efficacy[74, 75]. Moreover, enhancing MSC homing to specific tissue is likely to significantly reduce the number of cells that is required to achieve a therapeutic effect for disease, and might provide better outcomes for patients[76]. Our previous studies have demonstrated that systemic transplantation of hAD-MSCs can partly reduce ovarian injury and improve ovarian function in rats with chemotherapy-induced POI[8]. However, the homing rate of the systemically transplanted hAD-MSCs to 
POI ovaries was low, which might limit the efficiency of MSCs for POI treatment[37]. In this study, the results further demonstrated that the CXCR4 antagonist blocked SDF-1/CXCR4 axis and reduced the number of hAD-MSCs homing to the POI ovaries, which consistently reduced their efficacy in POI treatment. Thus, a better understanding of the underlying molecular mechanisms that direct the migration and homing of hAD-MSCs to chemotherapy-induced POI ovaries might be beneficial to exploring new ways to increase MSC homing rate and improve MSC transplantation efficacy, particularly in clinical applications. Our studies provide detailed evidence that SDF-1/CXCR4 axis partially mediates the migration and homing of systemically transplanted hAD-MSCs to chemotherapy-induced POI ovaries in rats, and PI3K/Akt signaling pathway might be involved in the migration and homing of hAD-MSCs mediated by SDF-1/CXCR4 axis. These findings may provide new evidence for the understanding of the molecular mechanisms involved in the migration and homing of hAD-MSCs to POI ovaries, and SDF1/CXCR4 axis might be a potential target to explore for increasing MSC homing rate and improving the efficacy of MSC transplantation for POI treatment, which will be explored in our subsequent studies.

\section{Conclusions}

In conclusion, our results demonstrate that SDF-1/CXCR4 axis partially mediates the migration and homing of systemically transplanted hAD-MSCs to chemotherapy-induced POI ovaries in rats. Systemic transplantation of hAD-MSCs can reduce ovarian injury and improve ovarian function in rats with chemotherapy-induced POI, and the block of SDF-1/CXCR4 axis with CXCR4 antagonist can reduce the number of hAD-MSCs homing to the POI ovaries, and further reduce their efficacy in POI treatment. PI3K/Akt signaling pathway, synchronously activated with SDF-1/CXCR4 axis, together plays a crucial role in the migration and homing of transplanted hAD-MSCs to chemotherapy-induced POI ovaries, which implicates PI3K/Akt signaling pathway might be involved in the migration and homing of hAD-MSCs mediated by SDF-1/CXCR4 axis. These findings may provide new evidence for the understanding of the molecular mechanisms involved in the migration and homing of hAD-MSCs to POI ovaries. This is a novel mechanism of hAD-MSC homing to chemotherapy-induced POI ovaries, and SDF-1/CXCR4 axis seems to be a potential target to explore for improving MSC transplantation efficacy in POI treatment by attracting systemically transplanted MSCs to the injured POI ovaries.

\section{Abbreviations}

POI: Premature ovarian insufficiency

MSCs: Mesenchymal stem cells

hAD-MSCs: Human amnion-derived mesenchymal stem cells

BM-MSCs: Bone marrow-derived mesenchymal stem cells

SDF-1: Stromal cell-derived factor-1

Page 19/36 
CXCR4: CXC chemokine receptor 4

ERK1/2: p44/p42 extracellular signal-regulated kinases

PI3K: Phosphatidylinositol-3-kinase

L-DMEM: Dulbecco's modified eagle medium low-glucose

FBS: Fetal bovine serum

CCK-8: Cell Counting Kit-8

AMH: Anti-Müllerian hormone

E2: Estradiol

FSH: Follicle-stimulating hormone

DAPI: 2-(4-amidinophenyl)-6-indolecarbamidine dihydrochloride

OD: Optical density

SD: Sprague-Dawley

HE: Hematoxylin and eosin

PBS: Phosphate-buffered saline

ELISA: Enzyme-linked immunosorbent assay

ADM: Adipogenic differentiation medium

ODM: Osteogenic differentiation medium

CDM: Chondrogenic differentiation medium

VEGF: Vascular endothelial growth factor

VEGFR2: Vascular endothelial growth factor receptor 2

IS: Immunoreactivity score

ISCT: International Society of Cell Therapy

GCs: Granulosa cells

FAK: Focal adhesion kinase 


\section{Declarations}

\section{Acknowledgements}

We thank the State Key Laboratory of Ultrasound Engineering in Medicine Co-Founded by Chongqing and the Ministry of Science and Technology, Chongqing Key Laboratory of Biomedical Engineering and Laboratory of Stem Cell and Tissue Engineering of Chongqing Medical University for their technical assistance.

\section{Funding}

This work was supported by the National Natural Science Foundation of China (No.81671415), Natural Science Foundation of Chongqing (No. cstc2019jcyj-msxmX0850) and Chongqing medical scientific research project (Joint project of Chongqing Health Commission and Science and Technology Bureau) (No. 2021MSXM061)

\section{Availability of data and materials}

All data and materials are available in the manuscript.

\section{Authors' contributions}

Conceived and designed the experiments: Li Ling.

Literature search: Li Ling, Jiying Hou, Dandan Liu, Dongyuan Tang.

Performed the experiments: Li Ling, Jiying Hou, Dandan Liu, Dongyuan Tang, Yanqin Zhang, Qianru Zeng, Heng Pan, Ling Fan.

Analyzed the data: Li Ling, Jiying Hou, Qianru Zeng, Heng Pan, Ling Fan.

Helped perform the analysis with constructive discussions: Li Ling, Jiying Hou, Dandan Liu, Dongyuan Tang, Yanqin Zhang, Qianru Zeng, Heng Pan, Ling Fan.

Drafted the manuscript: Li Ling.

All authors read and approved the final manuscript.

\section{Authors' information}

Not applicable. 


\section{Ethics approval and consent to participate}

The research was in compliance with the Helsinki Declaration and approved by the Ethics Committee of the Second Affiliated Hospital of Chongqing Medical University. Written informed consent was obtained from all donors before collecting amnion. Animal experimental protocols were approved by the Ethics Committee of the Second Affiliated Hospital of Chongqing Medical University (permit number 2016-044).

\section{Consent for publication}

Not applicable.

\section{Competing interests}

All authors declare no financial competing interests.

All authors declare no non-financial competing interests.

\section{References}

1. European Society for Human R, Embryology Guideline Group on POI. Webber L, Davies M, Anderson $\mathrm{R}$, Bartlett J, et al. ESHRE Guideline: management of women with premature ovarian insufficiency. Human reproduction. 2016;31(5):926-37.

2. Meirow $D$, Nugent $D$. The effects of radiotherapy and chemotherapy on female reproduction. Hum Reprod Update. 2001;7(6):535-43.

3. Wang Z, Wang Y, Yang T, Li J, Yang X. Study of the reparative effects of menstrual-derived stem cells on premature ovarian failure in mice. Stem Cell Res Ther. 2017;8(1):11.

4. Liu J, Zhang H, Zhang Y, Li N, Wen Y, Cao F, et al. Homing and restorative effects of bone marrowderived mesenchymal stem cells on cisplatin injured ovaries in rats. Mol Cells. 2014;37(12):865-72.

5. Wang S, Yu L, Sun M, Mu S, Wang C, Wang D, et al. The therapeutic potential of umbilical cord mesenchymal stem cells in mice premature ovarian failure. BioMed research international. 2013;2013:690491.

6. Sasaki M, Abe R, Fujita Y, Ando S, Inokuma D, Shimizu H. Mesenchymal stem cells are recruited into wounded skin and contribute to wound repair by transdifferentiation into multiple skin cell type. Journal of immunology. 2008;180(4):2581-7.

7. Nauta AJ, Fibbe WE. Immunomodulatory properties of mesenchymal stromal cells. Blood. 2007;110(10):3499-506.

8. Ling L, Feng X, Wei T, Wang Y, Wang Y, Wang Z, et al. Human amnion-derived mesenchymal stem cell (hAD-MSC) transplantation improves ovarian function in rats with premature ovarian insufficiency (POI) at least partly through a paracrine mechanism. Stem Cell Res Ther. 2019;10(1):46. 
9. Ling $L$, Wei $T$, He L, Wang $Y$, Wang $Y$, Feng $X$, et al. Low-intensity pulsed ultrasound activates ERK1/2 and PI3K-Akt signalling pathways and promotes the proliferation of human amnion-derived mesenchymal stem cells. Cell proliferation. 2017;50(6).

10. Diaz-Prado S, Muinos-Lopez E, Hermida-Gomez T, Rendal-Vazquez ME, Fuentes-Boquete I, de Toro FJ, et al. Multilineage differentiation potential of cells isolated from the human amniotic membrane. Journal of cellular biochemistry. 2010;111(4):846-57.

11. Ling L, Feng X, Wei T, Wang Y, Wang Y, Zhang W, et al. Effects of low-intensity pulsed ultrasound (LIPUS)-pretreated human amnion-derived mesenchymal stem cell (hAD-MSC) transplantation on primary ovarian insufficiency in rats. Stem Cell Res Ther. 2017;8(1):283.

12. Karp JM, Leng Teo GS. Mesenchymal stem cell homing: the devil is in the details. Cell stem cell. 2009;4(3):206-16.

13. Sohni A, Verfaillie CM. Mesenchymal stem cells migration homing and tracking. Stem cells international. 2013;2013:130763.

14. Chen J, Jiang J, Wang W, Qin J, Chen J, Chen W, et al. Low intensity pulsed ultrasound promotes the migration of bone marrow- derived mesenchymal stem cells via activating FAK-ERK1/2 signalling pathway. Artificial cells nanomedicine biotechnology. 2019;47(1):3603-13.

15. Croft AS, Illien-Junger S, Grad S, Guerrero J, Wangler S, Gantenbein B. The Application of Mesenchymal Stromal Cells and Their Homing Capabilities to Regenerate the Intervertebral Disc. Int J Mol Sci. 2021;22(7).

16. Belema-Bedada F, Uchida S, Martire A, Kostin S, Braun T. Efficient homing of multipotent adult mesenchymal stem cells depends on FROUNT-mediated clustering of CCR2. Cell stem cell. 2008;2(6):566-75.

17. Si YL, Zhao YL, Hao HJ, Fu XB, Han WD. MSCs: Biological characteristics, clinical applications and their outstanding concerns. Ageing Res Rev. 2011;10(1):93-103.

18. Teicher BA, Fricker SP. CXCL12 (SDF-1)/CXCR4 pathway in cancer. Clin Cancer Res. 2010;16(11):2927-31.

19. Ghadge SK, Muhlstedt S, Ozcelik C, Bader M. SDF-1alpha as a therapeutic stem cell homing factor in myocardial infarction. Pharmacol Ther. 2011;129(1):97-108.

20. Bonig H, Priestley GV, Papayannopoulou T. Hierarchy of molecular-pathway usage in bone marrow homing and its shift by cytokines. Blood. 2006;107(1):79-86.

21. Xue L, Wang J, Wang W, Yang Z, Hu Z, Hu M, et al. The effect of stromal cell-derived factor 1 in the migration of neural stem cells. Cell Biochem Biophys. 2014;70(3):1609-16.

22. Kucia M, Dawn B, Hunt G, Guo Y, Wysoczynski M, Majka M, et al. Cells expressing early cardiac markers reside in the bone marrow and are mobilized into the peripheral blood after myocardial infarction. Circ Res. 2004;95(12):1191-9.

23. Sordi V, Malosio ML, Marchesi F, Mercalli A, Melzi R, Giordano T, et al. Bone marrow mesenchymal stem cells express a restricted set of functionally active chemokine receptors capable of promoting migration to pancreatic islets. Blood. 2005;106(2):419-27. 
24. Ma J, Liu N, Yi B, Zhang X, Gao BB, Zhang Y, et al. Transplanted hUCB-MSCs migrated to the damaged area by SDF-1/CXCR4 signaling to promote functional recovery after traumatic brain injury in rats. Neurol Res. 2015;37(1):50-6.

25. Xiao Ling K, Peng L, Jian Feng Z, Wei C, Wei Yan Y, Nan S, et al. Stromal Derived Factor-1/CXCR4 Axis Involved in Bone Marrow Mesenchymal Stem Cells Recruitment to Injured Liver. Stem Cells Int. 2016;2016:8906945.

26. Xiu G, Li X, Yin Y, Li J, Li B, Chen X, et al. SDF-1/CXCR4 Augments the Therapeutic Effect of Bone Marrow Mesenchymal Stem Cells in the Treatment of Lipopolysaccharide-Induced Liver Injury by Promoting Their Migration Through PI3K/Akt Signaling Pathway. Cell Transplant. 2020;29:963689720929992.

27. Zhang H, Li X, Li J, Zhong L, Chen X, Chen S. SDF-1 mediates mesenchymal stem cell recruitment and migration via the SDF-1/CXCR4 axis in bone defect. J Bone Miner Metab. 2021;39(2):126-38.

28. Peyvandi AA, Roozbahany NA, Peyvandi H, Abbaszadeh HA, Majdinasab N, Faridan M, et al. Critical role of SDF-1/CXCR4 signaling pathway in stem cell homing in the deafened rat cochlea after acoustic trauma. Neural Regen Res. 2018;13(1):154-60.

29. Askari AT, Unzek S, Popovic ZB, Goldman CK, Forudi F, Kiedrowski M, et al. Effect of stromal-cellderived factor 1 on stem-cell homing and tissue regeneration in ischaemic cardiomyopathy. Lancet. 2003;362(9385):697-703.

30. Ji JF, He BP, Dheen ST, Tay SS. Interactions of chemokines and chemokine receptors mediate the migration of mesenchymal stem cells to the impaired site in the brain after hypoglossal nerve injury. Stem Cells. 2004;22(3):415-27.

31. Cheng X, Wang H, Zhang X, Zhao S, Zhou Z, Mu X, et al. The Role of SDF-1/CXCR4/CXCR7 in Neuronal Regeneration after Cerebral Ischemia. Front Neurosci. 2017;11:590.

32. Kawaguchi N, Zhang TT, Nakanishi T. Involvement of CXCR4 in Normal and Abnormal Development. Cells. 2019;8(2).

33. Chen J, Crawford R, Chen C, Xiao Y. The key regulatory roles of the PI3K/Akt signaling pathway in the functionalities of mesenchymal stem cells and applications in tissue regeneration. Tissue Eng Part $B$ Rev. 2013;19(6):516-28.

34. Li M, Sun X, Ma L, Jin L, Zhang W, Xiao M, et al. SDF-1/CXCR4 axis induces human dental pulp stem cell migration through FAK/PI3K/Akt and GSK3beta/beta-catenin pathways. Sci Rep. 2017;7:40161.

35. Li L, Lim RZL, Lee LSU, Chew NSY. HIV glycoprotein gp120 enhances mesenchymal stem cell migration by upregulating CXCR4 expression. Biochim Biophys Acta Gen Subj. 2018;1862(8):1790800.

36. Liu X, Duan B, Cheng Z, Jia X, Mao L, Fu H, et al. SDF-1/CXCR4 axis modulates bone marrow mesenchymal stem cell apoptosis, migration and cytokine secretion. Protein Cell. 2011;2(10):84554.

37. Feng X, Ling L, Zhang W, Liu X, Wang Y, Luo Y, et al. Effects of Human Amnion-Derived Mesenchymal Stem Cell (hAD-MSC) Transplantation In Situ on Primary Ovarian Insufficiency in SD Rats. 
Reproductive sciences. 2020.

38. De Flora S, Ferguson LR. Overview of mechanisms of cancer chemopreventive agents. Mutat Res. 2005;591(1-2):8-15.

39. Bedoschi G, Navarro PA, Oktay K. Chemotherapy-induced damage to ovary: mechanisms and clinical impact. Future Oncol. 2016;12(20):2333-44.

40. Meirow D, Dor J, Kaufman B, Shrim A, Rabinovici J, Schiff E, et al. Cortical fibrosis and blood-vessels damage in human ovaries exposed to chemotherapy. Potential mechanisms of ovarian injury. Hum Reprod. 2007;22(6):1626-33.

41. Wang X, Wang C, Gou W, Xu X, Wang Y, Wang A, et al. The optimal time to inject bone mesenchymal stem cells for fracture healing in a murine model. Stem Cell Res Ther. 2018;9(1):272.

42. Zhong J, Rajagopalan S. Dipeptidyl Peptidase-4 Regulation of SDF-1/CXCR4 Axis: Implications for Cardiovascular Disease. Front Immunol. 2015;6:477.

43. Jaszczynska-Nowinka K, Rucinski M, Ziolkowska A, Markowska A, Malendowicz LK. Expression of SDF-1 and CXCR4 transcript variants and CXCR7 in epithelial ovarian cancer. Oncol Lett. 2014;7(5):1618-24.

44. Scotton CJ, Wilson JL, Milliken D, Stamp G, Balkwill FR. Epithelial cancer cell migration: a role for chemokine receptors? Cancer Res. 2001;61(13):4961-5.

45. Su L, Hu Z, Yang YG. Role of CXCR4 in the progression and therapy of acute leukaemia. Cell Prolif. 2021:e13076.

46. Jin W, Liang X, Brooks A, Futrega K, Liu X, Doran MR, et al. Modelling of the SDF-1/CXCR4 regulated in vivo homing of therapeutic mesenchymal stem/stromal cells in mice. PeerJ. 2018;6:e6072.

47. Tang Q, Luo C, Lu B, Fu Q, Yin H, Qin Z, et al. Thermosensitive chitosan-based hydrogels releasing stromal cell derived factor-1 alpha recruit MSC for corneal epithelium regeneration. Acta Biomater. 2017;61:101-13.

48. Pagella P, Nombela-Arrieta C, Mitsiadis TA. Distinct Expression Patterns of Cxcl12 in Mesenchymal Stem Cell Niches of Intact and Injured Rodent Teeth. Int J Mol Sci. 2021;22(6).

49. Yu J, Kim HM, Kim KP, Son Y, Kim MS, Park KS. Ceramide kinase regulates the migration of bone marrow-derived mesenchymal stem cells. Biochem Biophys Res Commun. 2019;508(2):361-7.

50. Werner L, Guzner-Gur H, Dotan I. Involvement of CXCR4/CXCR7/CXCL12 Interactions in Inflammatory bowel disease. Theranostics. 2013;3(1):40-6.

51. Karin N. The multiple faces of CXCL12 (SDF-1alpha) in the regulation of immunity during health and disease. J Leukoc Biol. 2010;88(3):463-73.

52. Jiang Q, Song P, Wang E, Li J, Hu S, Zhang H. Remote ischemic postconditioning enhances cell retention in the myocardium after intravenous administration of bone marrow mesenchymal stromal cells. J Mol Cell Cardiol. 2013;56:1-7.

53. Abbott JD, Huang Y, Liu D, Hickey R, Krause DS, Giordano FJ. Stromal cell-derived factor-1alpha plays a critical role in stem cell recruitment to the heart after myocardial infarction but is not sufficient to 
induce homing in the absence of injury. Circulation. 2004;110(21):3300-5.

54. Kitaori T, Ito H, Schwarz EM, Tsutsumi R, Yoshitomi H, Oishi S, et al. Stromal cell-derived factor $1 / C X C R 4$ signaling is critical for the recruitment of mesenchymal stem cells to the fracture site during skeletal repair in a mouse model. Arthritis rheumatism. 2009;60(3):813-23.

55. Luo Q, Yin N, Zhang L, Yuan W, Zhao W, Luan X, et al. Role of SDF-1/CXCR4 and cytokines in the development of ovary injury in chemotherapy drug induced premature ovarian failure mice. Life Sci. 2017;179:103-9.

56. Liekens S, Schols D, Hatse S. CXCL12-CXCR4 axis in angiogenesis, metastasis and stem cell mobilization. Curr Pharm Des. 2010;16(35):3903-20.

57. Kavanagh DP, Kalia N. Hematopoietic stem cell homing to injured tissues. Stem Cell Rev Rep. 2011;7(3):672-82.

58. Strioga M, Viswanathan S, Darinskas A, Slaby O, Michalek J. Same or not the same? Comparison of adipose tissue-derived versus bone marrow-derived mesenchymal stem and stromal cells. Stem Cells Dev. 2012;21(14):2724-52.

59. Rombouts WJ, Ploemacher RE. Primary murine MSC show highly efficient homing to the bone marrow but lose homing ability following culture. Leukemia. 2003;17(1):160-70.

60. Won YW, Patel AN, Bull DA. Cell surface engineering to enhance mesenchymal stem cell migration toward an SDF-1 gradient. Biomaterials. 2014;35(21):5627-35.

61. de Assis-Ferreira A, Saldanha-Gama R, de Brito NM, Renovato-Martins M, Simoes RL, Barja-Fidalgo C, et al. Obesity enhances the recruitment of mesenchymal stem cells to visceral adipose tissue. $\mathrm{J} \mathrm{Mol}$ Endocrinol. 2021;67(1):15-26.

62. Petit I, Jin D, Rafii S. The SDF-1-CXCR4 signaling pathway: a molecular hub modulating neoangiogenesis. Trends Immunol. 2007;28(7):299-307.

63. Togel F, Isaac J, Hu Z, Weiss K, Westenfelder C. Renal SDF-1 signals mobilization and homing of CXCR4-positive cells to the kidney after ischemic injury. Kidney Int. 2005;67(5):1772-84.

64. Chang LT, Yuen CM, Sun CK, Wu CJ, Sheu JJ, Chua S, et al. Role of stromal cell-derived factor-1alpha, level and value of circulating interleukin-10 and endothelial progenitor cells in patients with acute myocardial infarction undergoing primary coronary angioplasty. Circ J. 2009;73(6):1097-104.

65. Segers VF, Tokunou T, Higgins LJ, MacGillivray C, Gannon J, Lee RT. Local delivery of proteaseresistant stromal cell derived factor- 1 for stem cell recruitment after myocardial infarction. Circulation. 2007;116(15):1683-92.

66. Huang X, Guo B, Liu S, Wan J, Broxmeyer HE. Neutralizing negative epigenetic regulation by HDAC5 enhances human haematopoietic stem cell homing and engraftment. Nat Commun. 2018;9(1):2741.

67. Wang Y, Deng Y, Zhou GQ. SDF-1alpha/CXCR4-mediated migration of systemically transplanted bone marrow stromal cells towards ischemic brain lesion in a rat model. Brain Res. 2008;1195:10412. 
68. Wei FY, Leung KS, Li G, Qin J, Chow SK, Huang S, et al. Low intensity pulsed ultrasound enhanced mesenchymal stem cell recruitment through stromal derived factor-1 signaling in fracture healing. PLoS One. 2014;9(9):e106722.

69. Song YL, Jiang H, Jiang NG, Jin YM, Zeng TT. Mesenchymal Stem Cell-Platelet Aggregates Increased in the Peripheral Blood of Patients with Acute Myocardial Infarction and Might Depend on the Stromal Cell-Derived Factor 1/CXCR4 Axis. Stem Cells Dev. 2019;28(24):1607-19.

70. Kucia M, Jankowski K, Reca R, Wysoczynski M, Bandura L, Allendorf DJ, et al. CXCR4-SDF-1 signalling, locomotion, chemotaxis and adhesion. J Mol Histol. 2004;35(3):233-45.

71. Wong D, Korz W. Translating an Antagonist of Chemokine Receptor CXCR4: from bench to bedside. Clin Cancer Res. 2008;14(24):7975-80.

72. Demirci S, Dogan A, Apdik H, Tuysuz EC, Gulluoglu S, Bayrak OF, et al. Cytoglobin inhibits migration through PI3K/AKT/mTOR pathway in fibroblast cells. Mol Cell Biochem. 2018;437(1-2):133-42.

73. Chen D, Xia Y, Zuo K, Wang Y, Zhang S, Kuang D, et al. Crosstalk between SDF-1/CXCR4 and SDF1/CXCR7 in cardiac stem cell migration. Sci Rep. 2015;5:16813.

74. Xiao GY, Liu IH, Cheng CC, Chang CC, Lee YH, Cheng WT, et al. Amniotic fluid stem cells prevent follicle atresia and rescue fertility of mice with premature ovarian failure induced by chemotherapy. PloS one. 2014;9(9):e106538.

75. Lai D, Wang F, Yao X, Zhang Q, Wu X, Xiang C. Human endometrial mesenchymal stem cells restore ovarian function through improving the renewal of germline stem cells in a mouse model of premature ovarian failure. Journal of translational medicine. 2015;13:155.

76. Walczak P, Zhang J, Gilad AA, Kedziorek DA, Ruiz-Cabello J, Young RG, et al. Dual-modality monitoring of targeted intraarterial delivery of mesenchymal stem cells after transient ischemia. Stroke. 2008;39(5):1569-74.

\section{Figures}



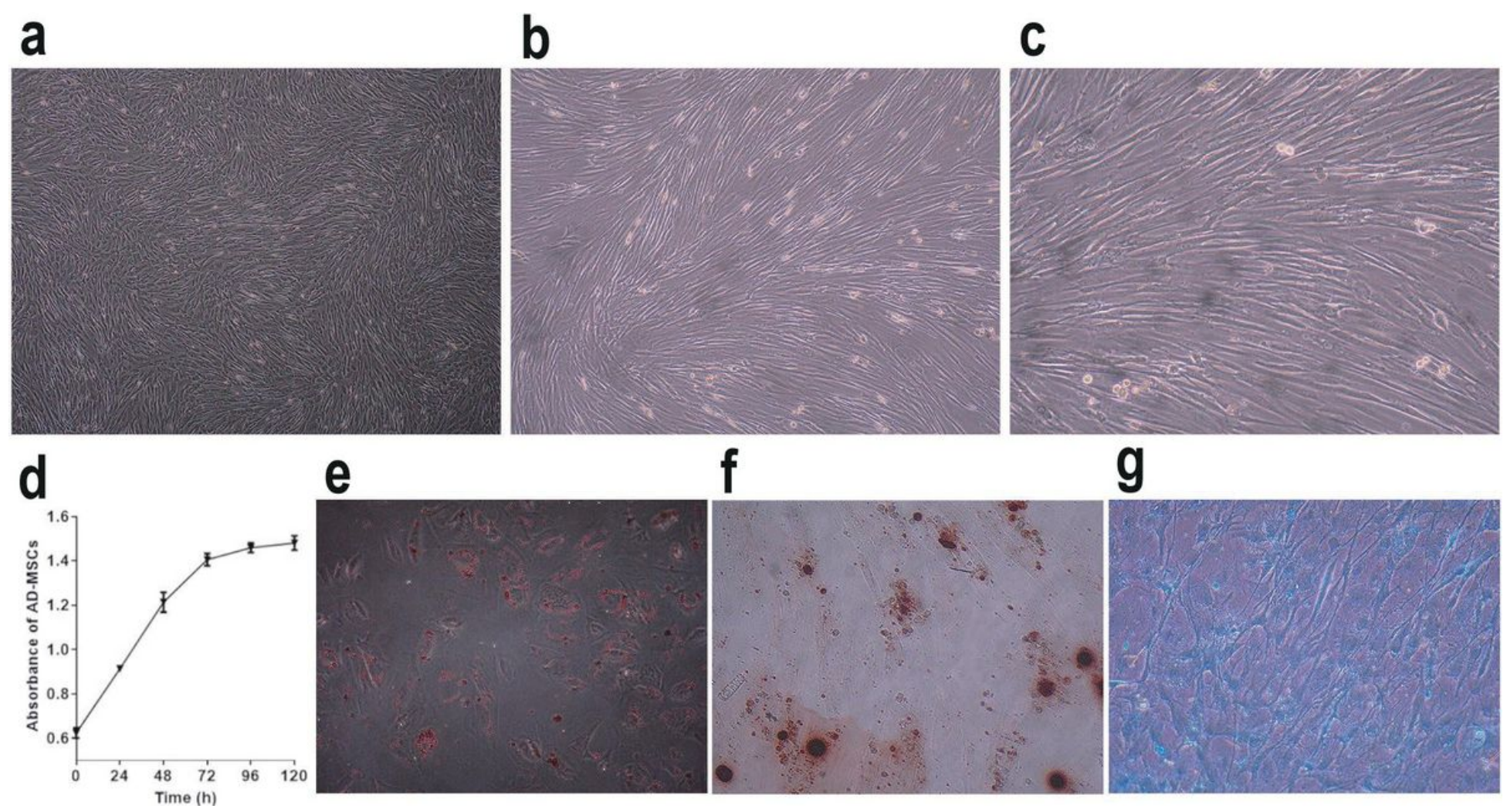

f

\section{g}
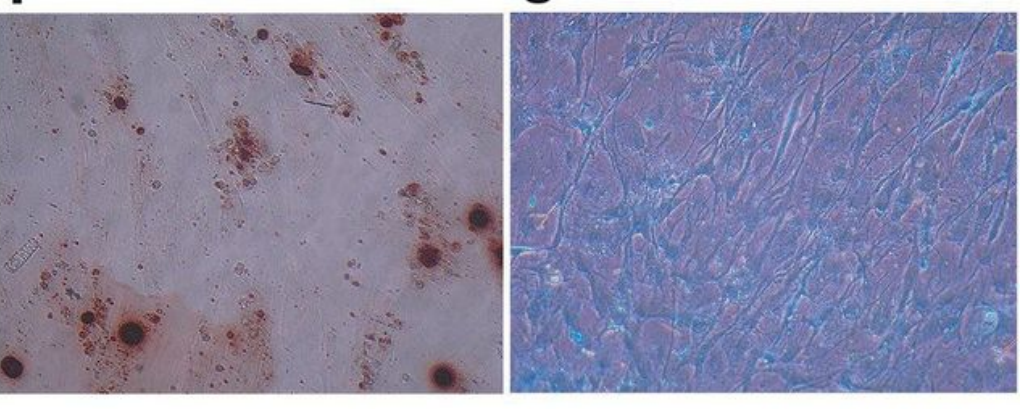

\section{Figure 1}

Characterization of hAD-MSCs. a-c Morphology of hAD-MSCs $(a \times 40, b \times 100, c \times 200)$. $d$ The growth curve of hAD-MSCs. e-g hAD-MSCs was able to differentiate into adipocytes $(e \times 200)$, osteoblasts $(f \times 200)$ and chondroblasts $(g \times 200)$. Lipid vacuoles in the cytoplasm were visualized in adipocytes, which were verified by Oil Red $\mathrm{O}$ staining (e). Abundant calcium deposits were visualized in osteoblasts, which were stained by Alizarin Red S staining (f). Cartilage-specific proteoglycans (g) were visualized in chondroblasts, which were stained by Alcian blue. Representative images are shown. 
a

b

CXCR4
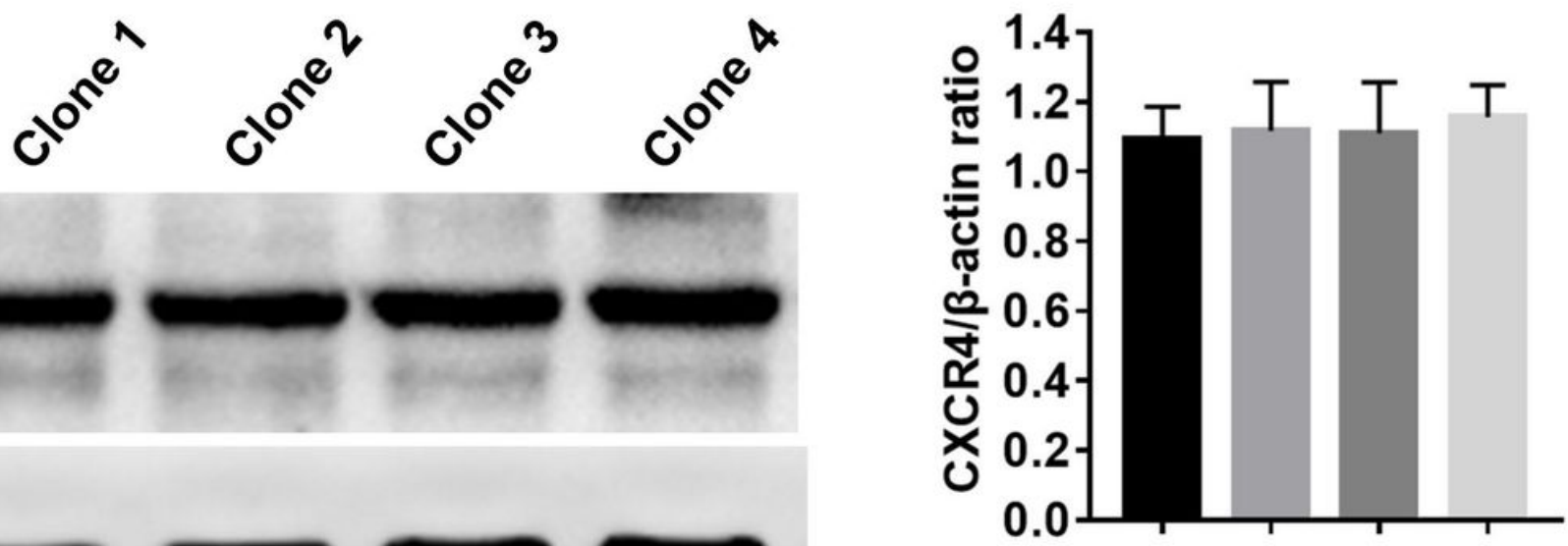

$\beta$-actin

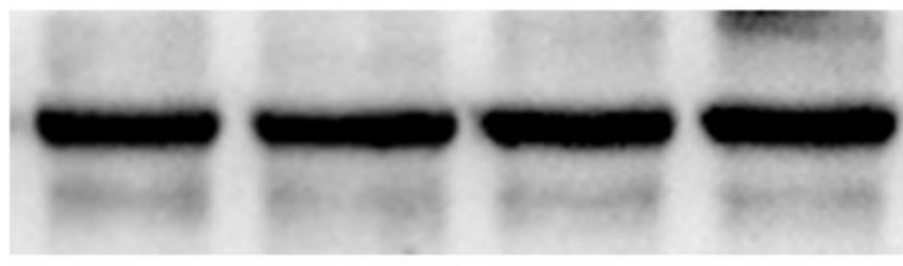

C

\section{Merge}

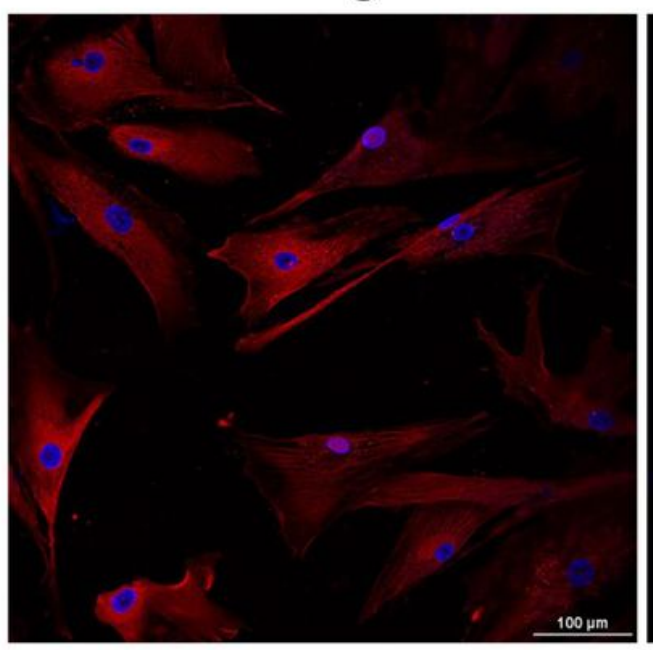

DAPI

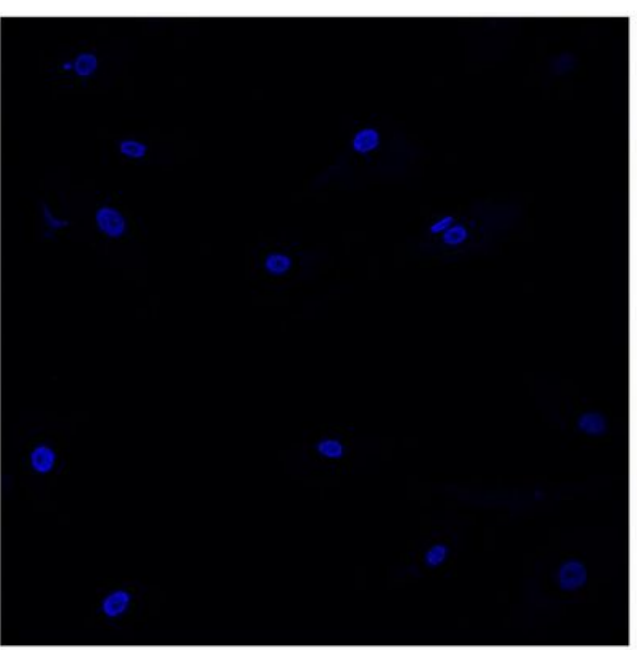

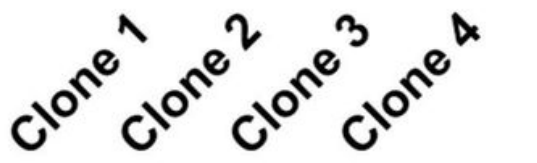

CXCR4

\section{Figure 2}

Expression of CXCR4 in hAD-MSCs. $a$, b Western blot assay was performed to detect the protein expression levels of CXCR4 in hAD-MSCs (a) and the CXCR4/ $\beta$-actin ratios were evaluated (b). C Immunofluorescence assay was performed to confirm the protein expression of CXCR4 in hAD-MSCs ( $\times 200)$. Representative images are shown. ${ }^{*} P<0.05$ and $* * P<0.01$. Scale bars $=100 \mu \mathrm{m}$. 
a
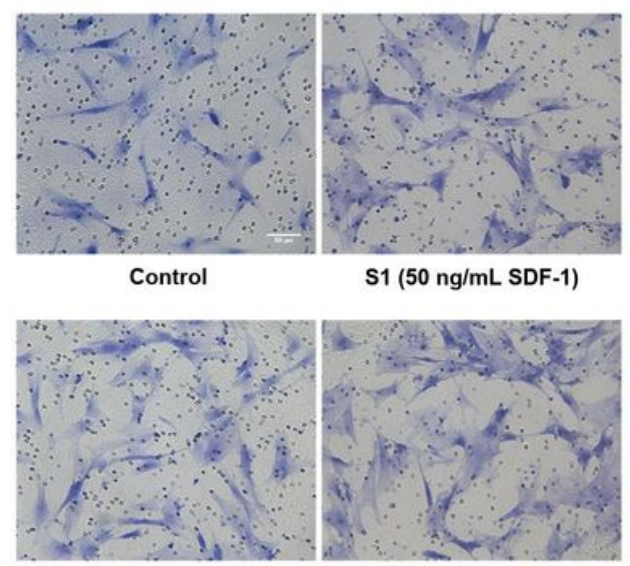

S2 (100 ng/mL SDF-1)

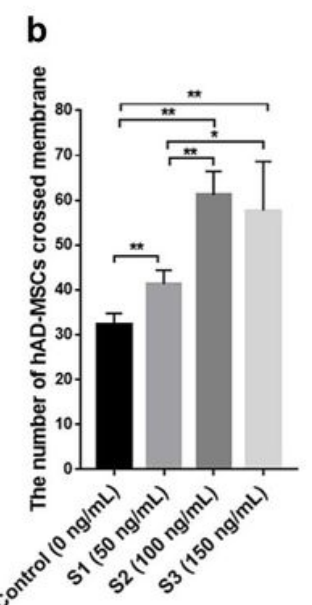

The concentration of SDF-1 (ng/mL) c

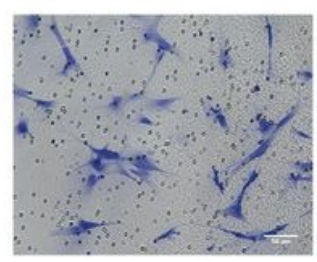

Control

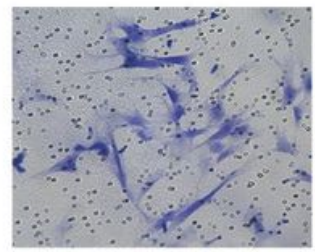

SDF-1+ AMD3100 d

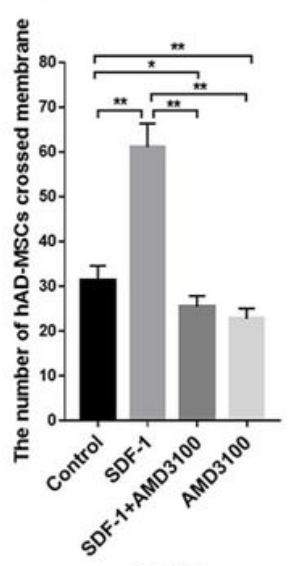

Groups

Figure 3

SDF-1 induces the migration of hAD-MSCs in vitro. $a, b$ The migration of hAD-MSCs was tested by transwell migration assay with $0,50,100$ or $150 \mathrm{ng} / \mathrm{mL}$ of SDF-1, in vitro $(\times 200)$. c, d The role of SDF$1 / C X C R 4$ axis in the migration of hAD-MSCs induced by SDF-1 was detected by transwell migration assay. The migration of hAD-MSCs was tested in the control, SDF-1, SDF-1+AMD3100 and AMD3100 groups ( $\times 200$ ). Representative images are shown. $N=6, * P<0.05$ and ${ }^{*} * P<0.01$. Scale bars $=50 \mu \mathrm{m}$.

a

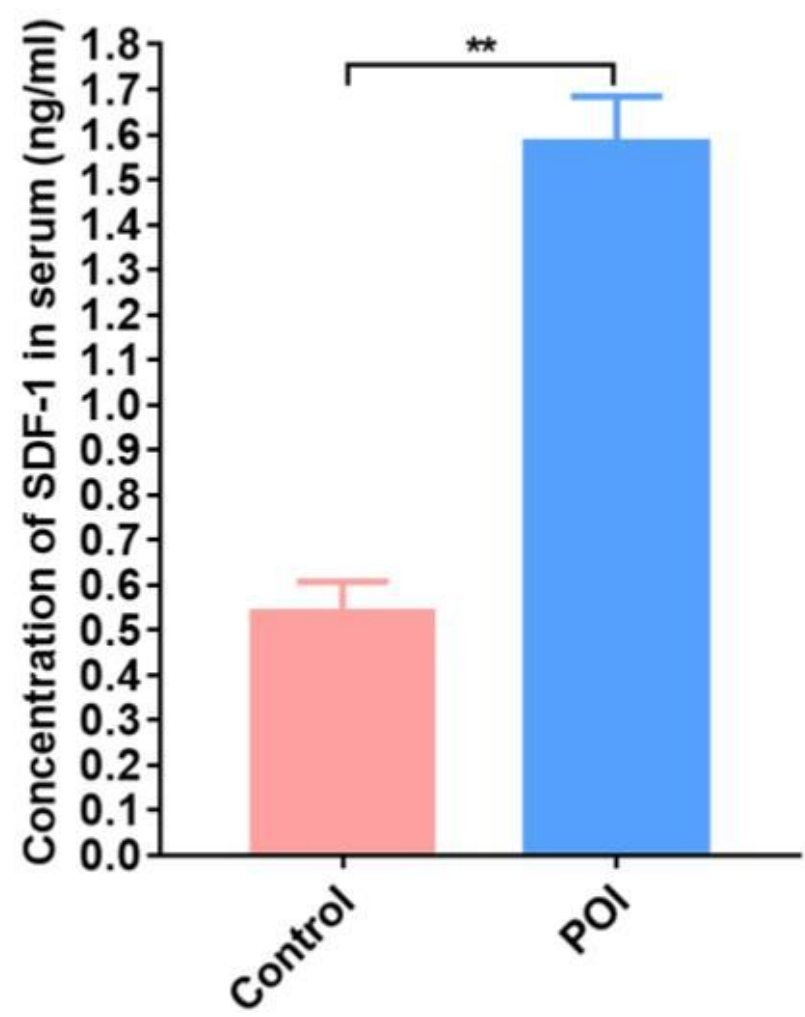

Groups b

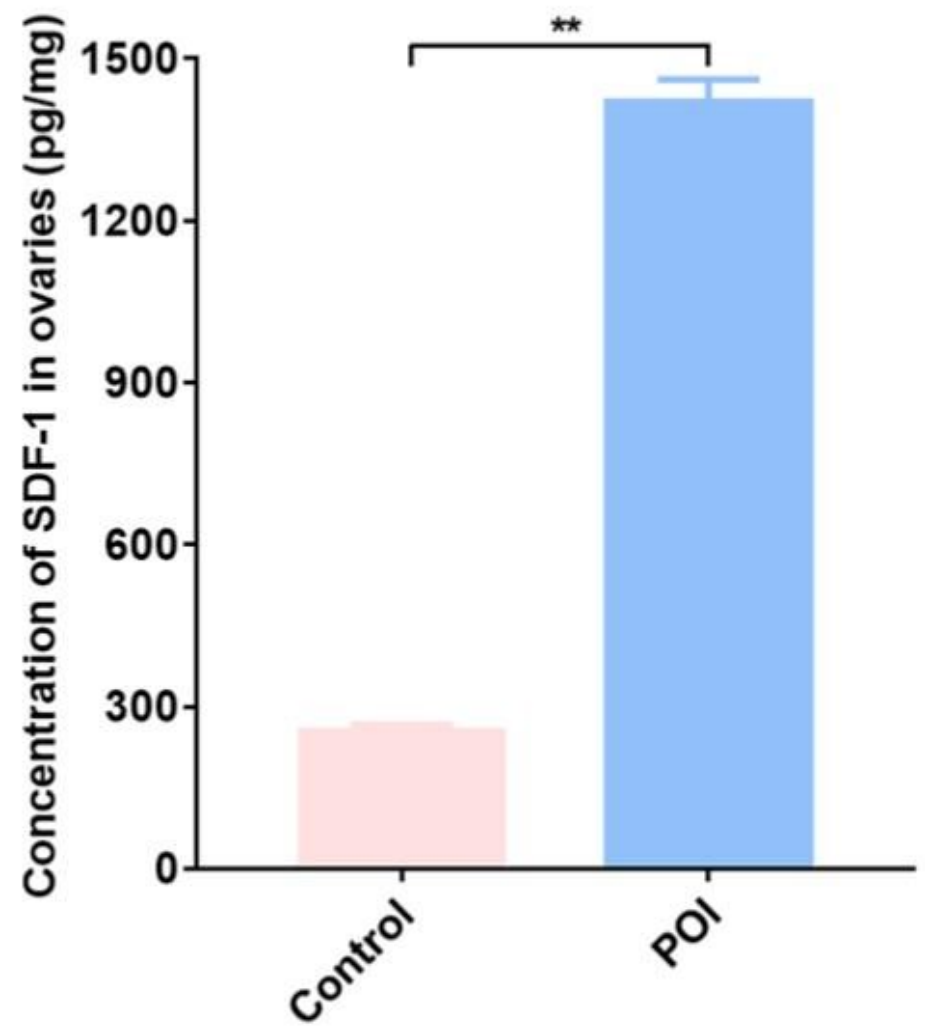

Groups

Figure 4 
Elevated levels of SDF-1 in serum and ovaries induced by chemotherapy in rats with POI. SDF-1 concentrations in the serum (a) and supernatant of homogenized ovarian tissue (b) in the control and POI groups were measured by ELISA assay. ${ }^{*} \mathrm{P}<0.05$ and $* * \mathrm{P}<0.01$.

a

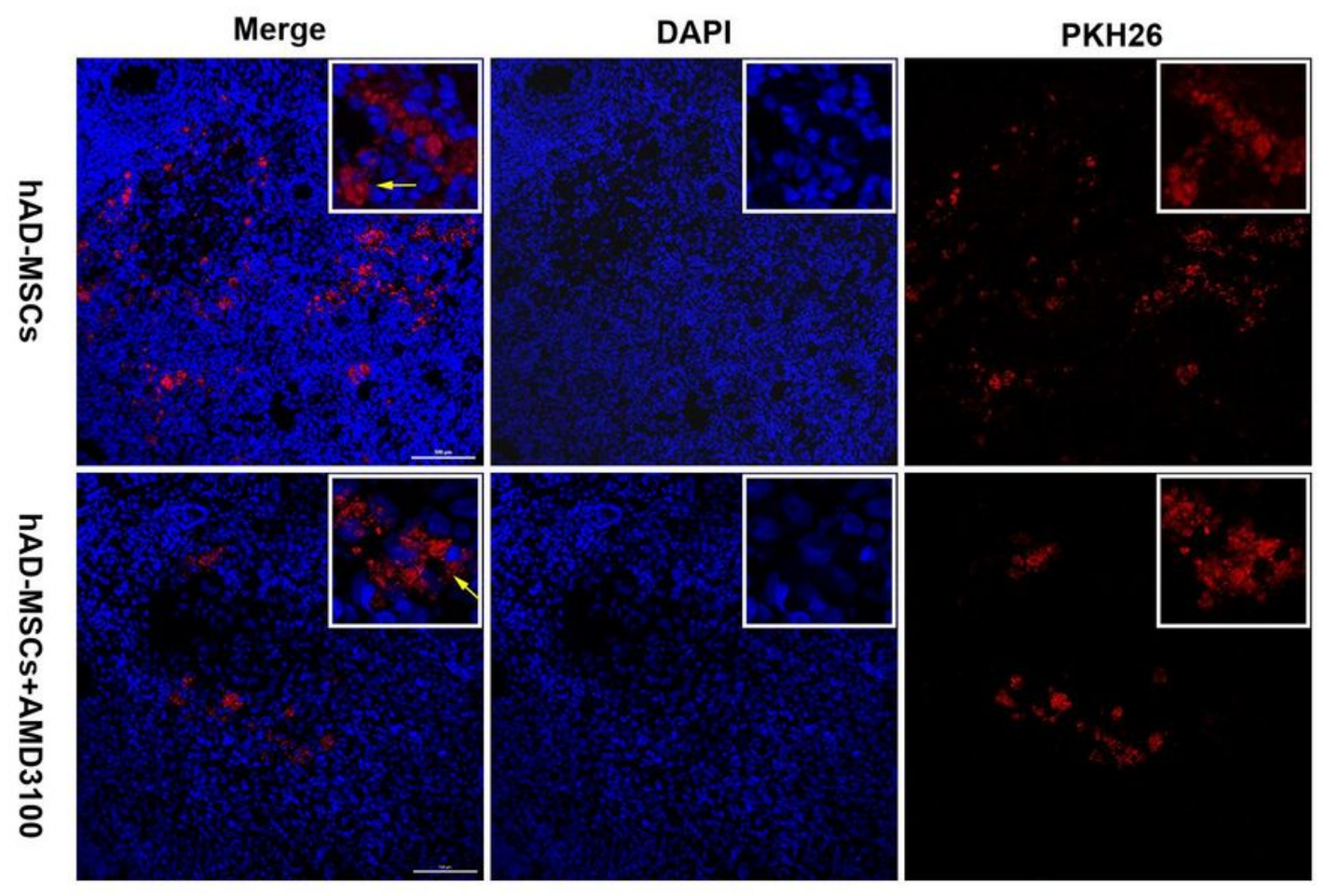

b

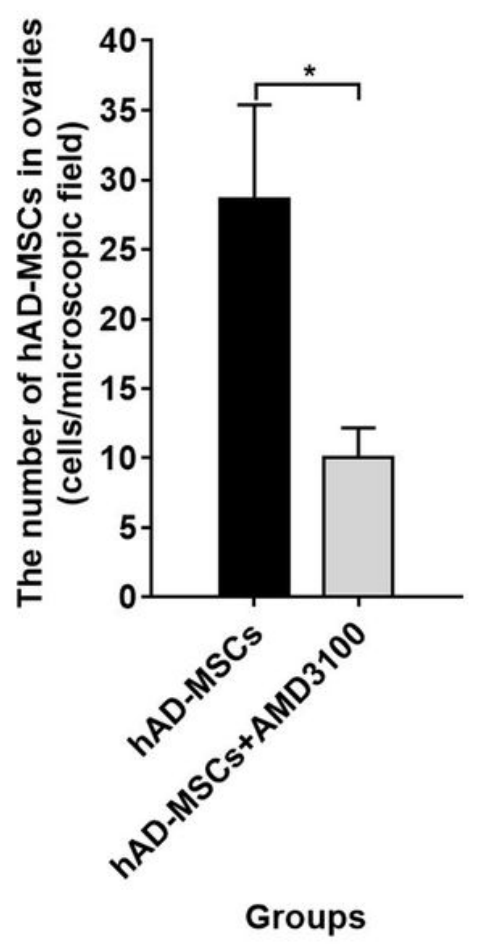

Figure 5

SDF-1/CXCR4 axis is involved in the homing of hAD-MSCs to chemotherapy-induced POI ovaries in rats. a Transplanted PKH26-labeled hAD-MSCs were observed in ovaries at $24 \mathrm{~h}$ after cell transplantation in the hAD-MSCs and hAD-MSCs+AMD3100 groups by confocal microscopy analysis $(\times 200$ and $\times 800)$. b The number of hAD-MSCs was counted and compared in the hAD-MSCs and hAD-MSCs+AMD3100 groups $(n=10)$. Representative images are shown. The yellow arrows indicate transplanted hAD-MSCs. *P $<0.05$. Scale bars $=100 \mu \mathrm{m}$. 
a

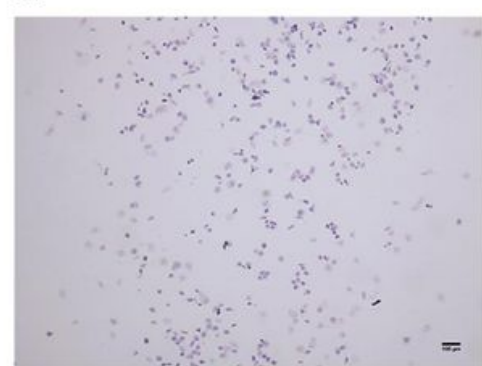

Proestrus

b

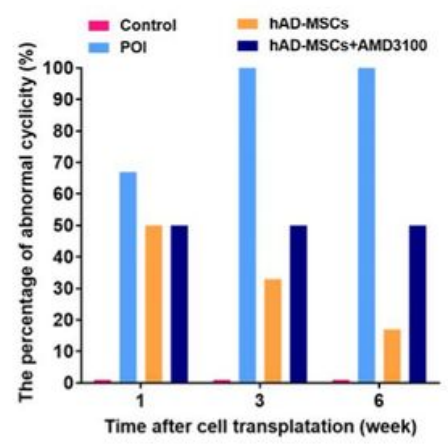

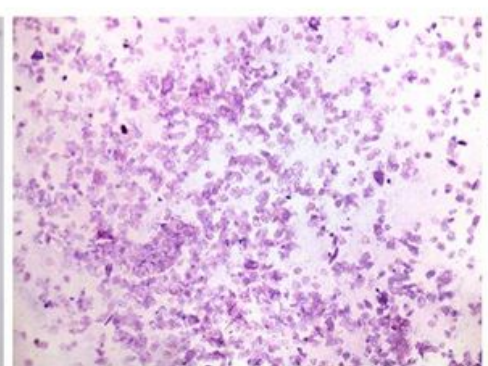

Estrus

C

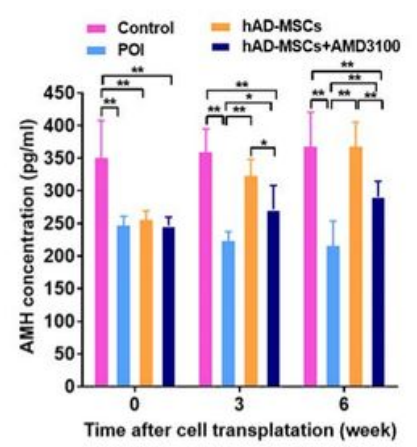

\section{Metestrus}

d

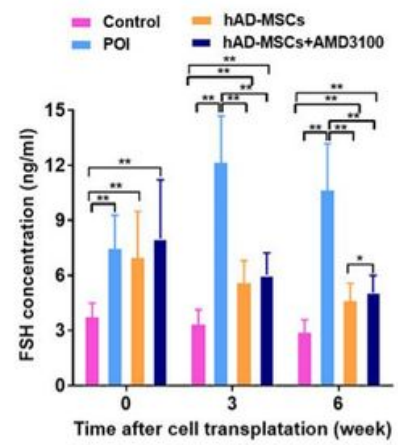

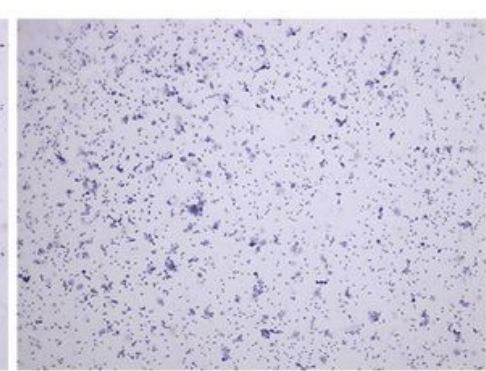

Diestrus

e

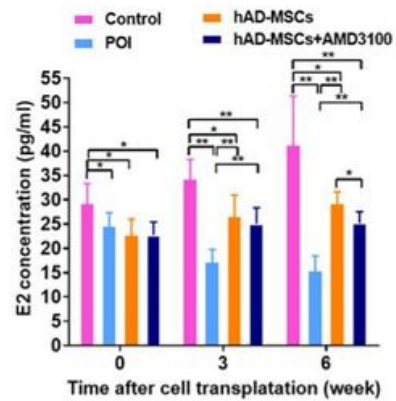

Figure 6

Effects of hAD-MSCs on ovarian function in rats with chemotherapy-induced POI after blocking SDF1/CXCR4 axis. a Estrous cycles of rats were observed. Regular estrous cycles consist of 4 sequential stages, proestrus, estrus, metestrus and diestrus $(\times 40)$. b The percentages of rats with abnormal cyclicity were detected at 1, 3, and 6 weeks after cell transplantation. c-e Serum levels of AMH (c), FSH (d) and E2 (e) were detected at 0, 3, 6 weeks after cell transplantation. Representative images are shown. ${ }^{*} \mathrm{P}<0.05$ and ${ }^{*} \mathrm{P}<0.01$. Scale bars $=100 \mu \mathrm{m}$. 

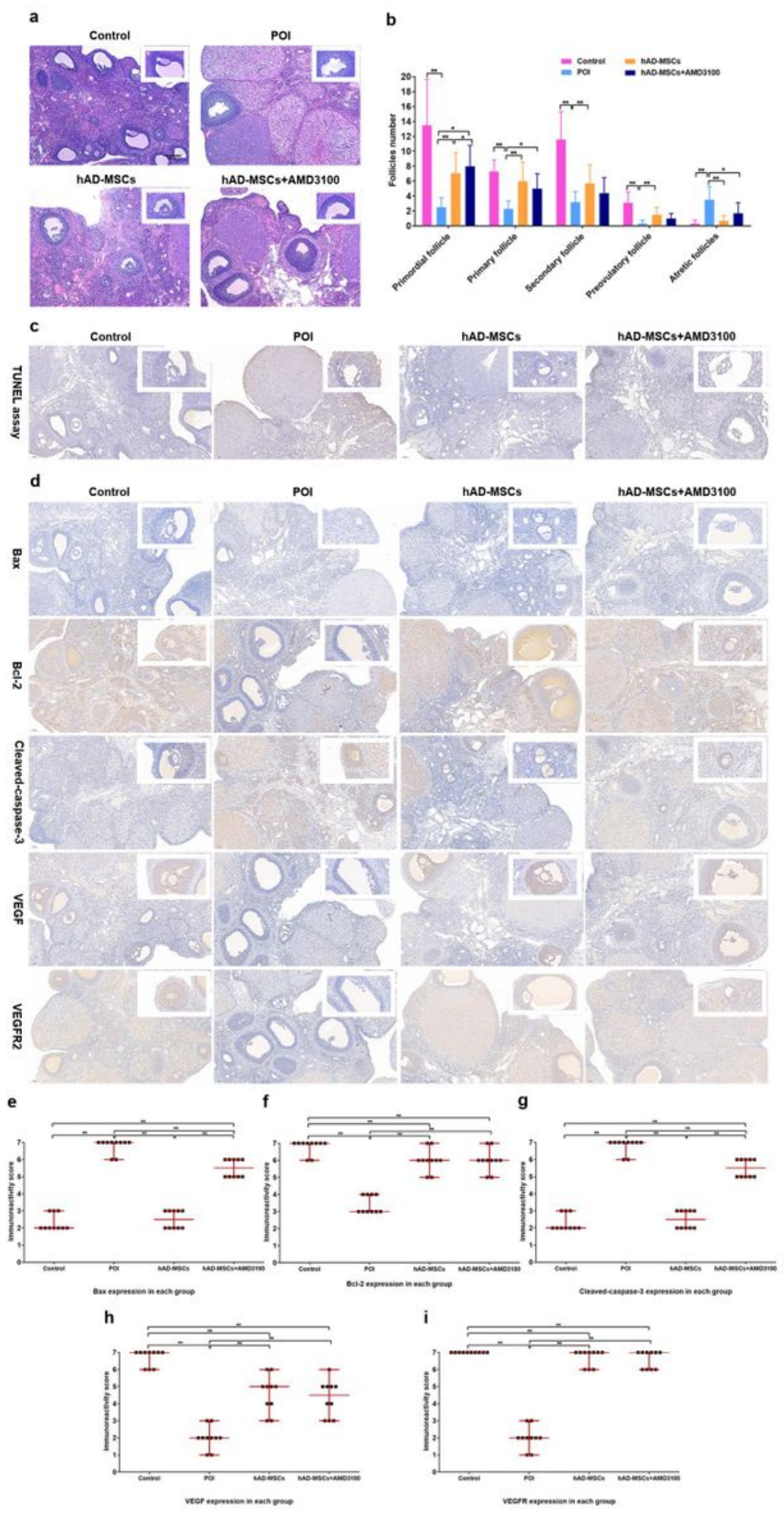

\section{Figure 7}

Effects of hAD-MSCs on ovarian injuries induced by chemotherapy in POI rats after blocking SDF1/CXCR4 axis. a Ovarian tissue changes were analyzed by HE staining $(\times 100$ and $\times 200)$. $b$ The number of follicles at different stages was counted and compared in the control, POI, hAD-MSCs and hADMSCs+AMD3100 groups $(n=10)$. c Ovarian granulosa cell $(G C)$ apoptosis was tested by TUNEL assay ( $\times 100$ and $\times 400)$. $d$ The expression levels of Bax, Bcl-2, cleaved-caspase-3, VEGF and VEGFR2 in the 
ovaries were detected by immunohistochemical staining $(\times 100$ and $\times 400)$. e-i Semiquantitative analyses of Bax, Bcl-2, cleaved-caspase-3, VEGF and VEGFR2 expression in the ovaries of each group were shown $(n=10)$. Each dot in graphs (e-i) represents the value across ten high-power fields (HPFs) randomly chosen from five sections in each group. The bars and error bars in graphs (e-i) indicate the medians and ranges, respectively. Brown cells represent immunostained cells. Representative images are shown. *P < 0.05 and $* * P<0.01$. Scale bars $=100 \mu \mathrm{m}$.

a

\section{Control SDF-1}

phospho-ERK1/2

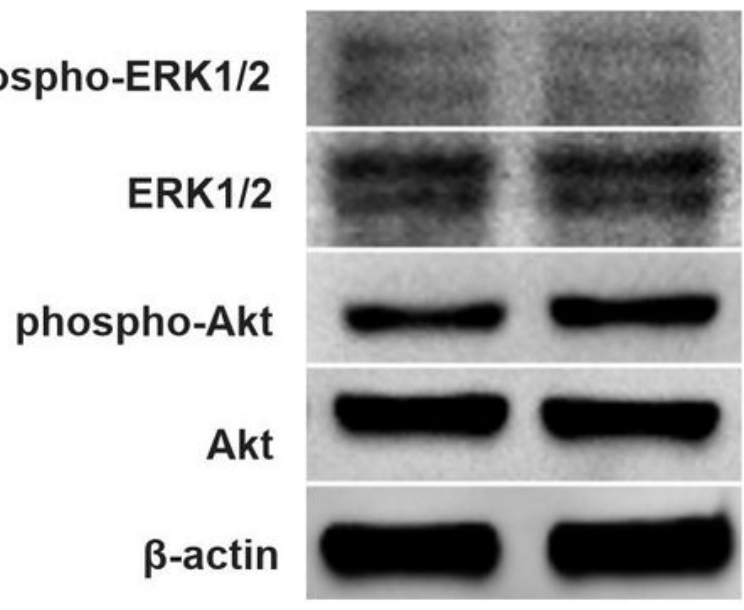

d b

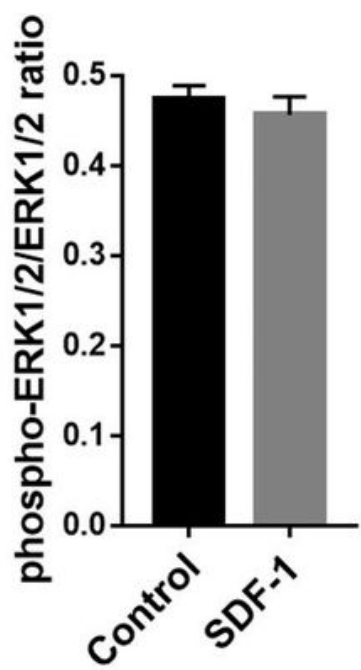

Groups
C

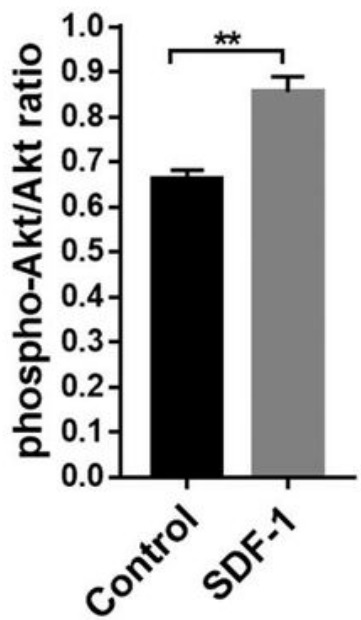

Groups

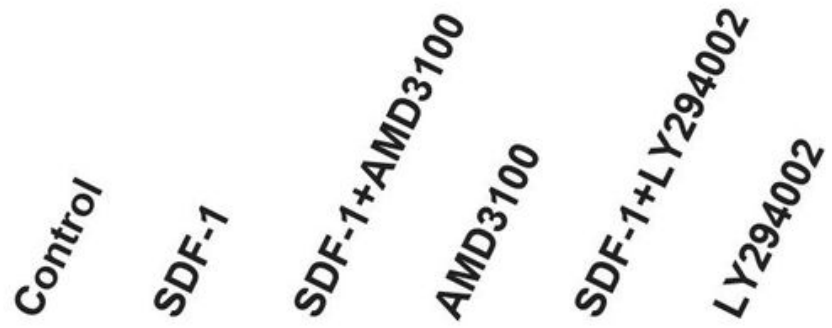

phospho-Akt

Akt

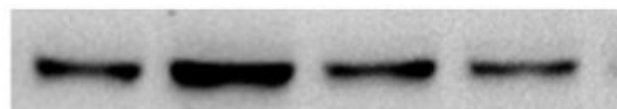


Activation of PI3K/Akt signaling pathway by SDF-1 in hAD-MSCs. a-c Effects of SDF-1 on the phosphorylation of ERK1/2 and Akt in hAD-MSCs were determined by Western blot analysis (a), and the phospho-ERK1/2/ERK1/2 and phospho-Akt/Akt ratios were evaluated (b, c). d, e Akt and phospho-Akt were analysed by Western blot after the pretreatment of hAD-MSCs with AMD3100 or LY294002 for 1 hour followed by the treatment of SDF-1 (d), and the phospho-Akt/Akt ratios were evaluated (e). ${ }^{*}<<0.05$ and $* * \mathrm{P}<0.01$.

\section{a}

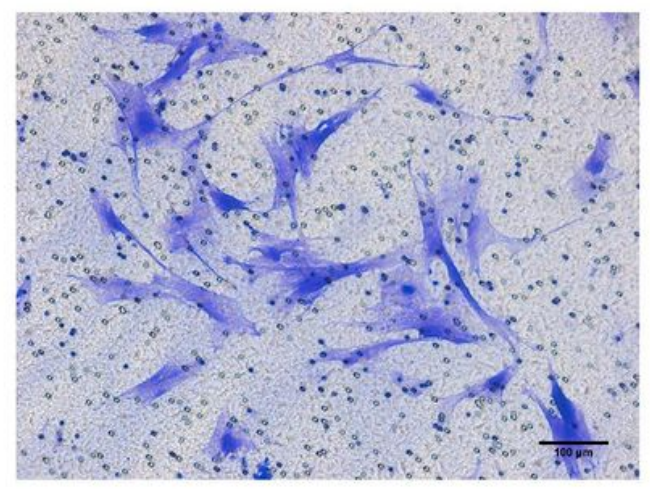

Control

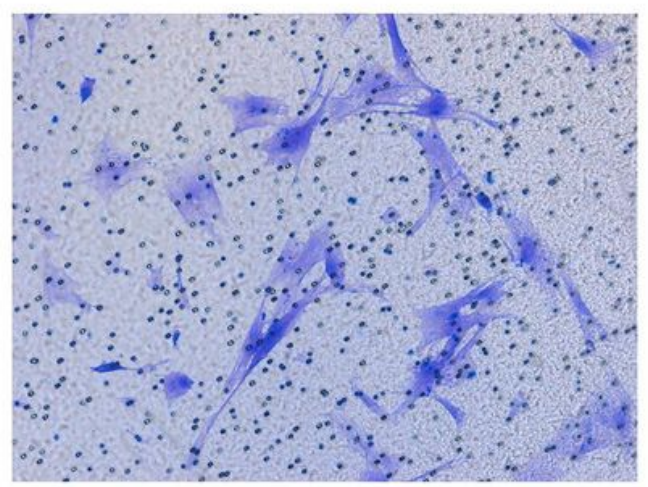

SDF-1+LY294002

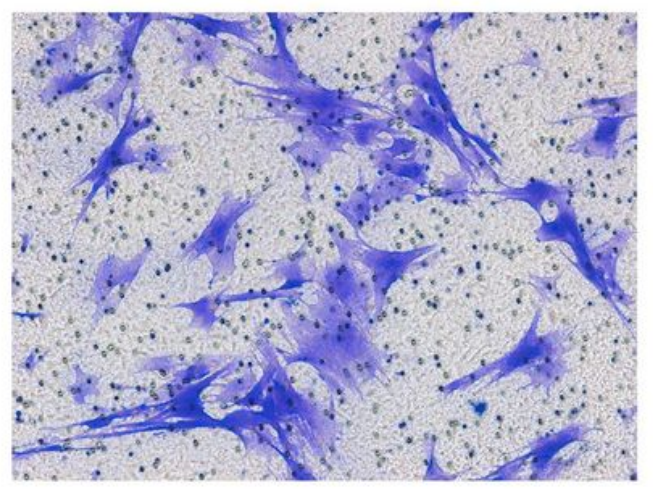

SDF-1

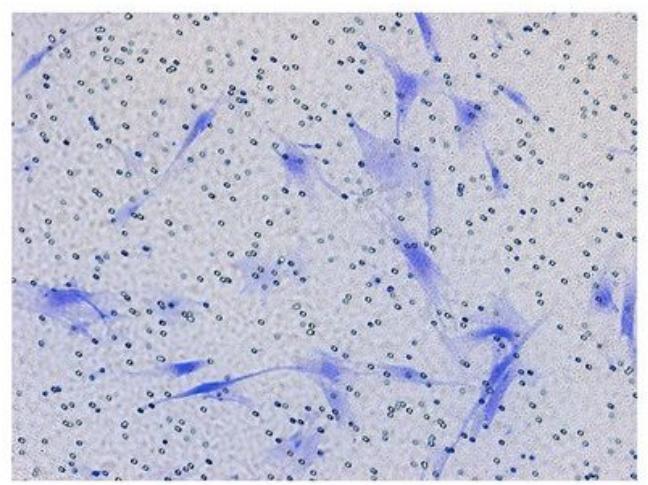

LY294002
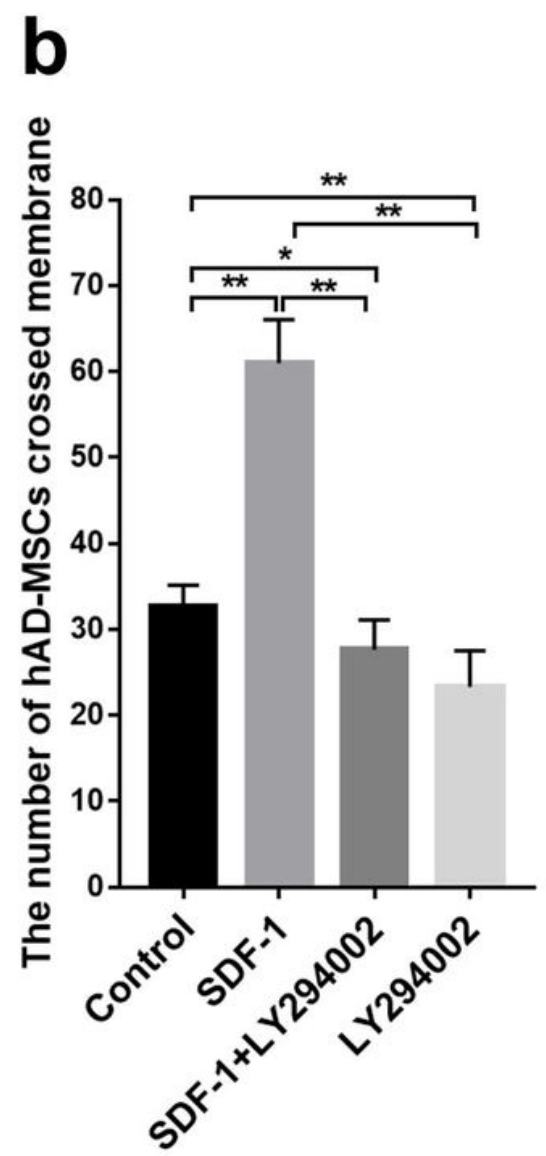

Groups

\section{Figure 9}

Effects of PI3K/Akt signaling pathway on the migration of hAD-MSCs mediated by SDF-1/CXCR4 axis. The migration of hAD-MSCs was tested by transwell migration assay (a) and migrated cells were counted (b) in the control, SDF-1, SDF-1+LY294002 and LY294002 groups under microscope $(\times 100)$. Representative images are shown. $N=6, * P<0.05$ and ${ }^{*} * P<0.01$. Scale bars $=100 \mu \mathrm{m}$. 
a
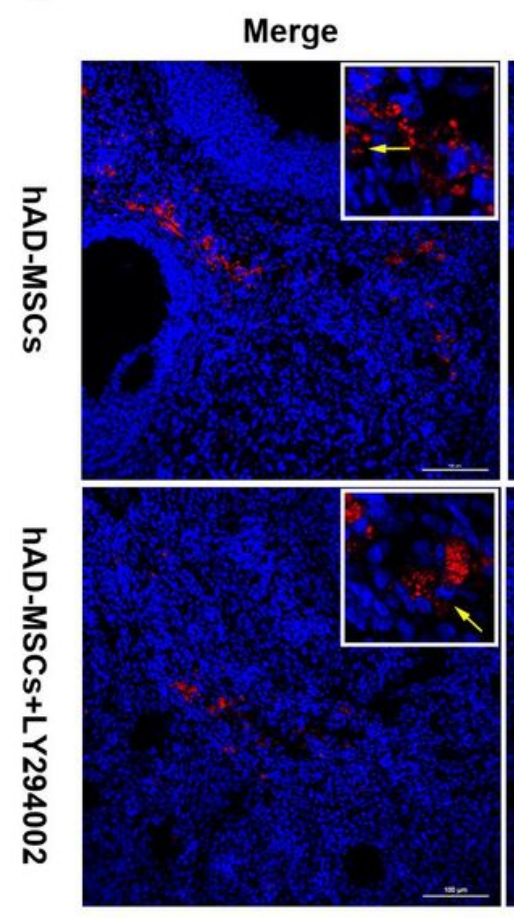

DAPI
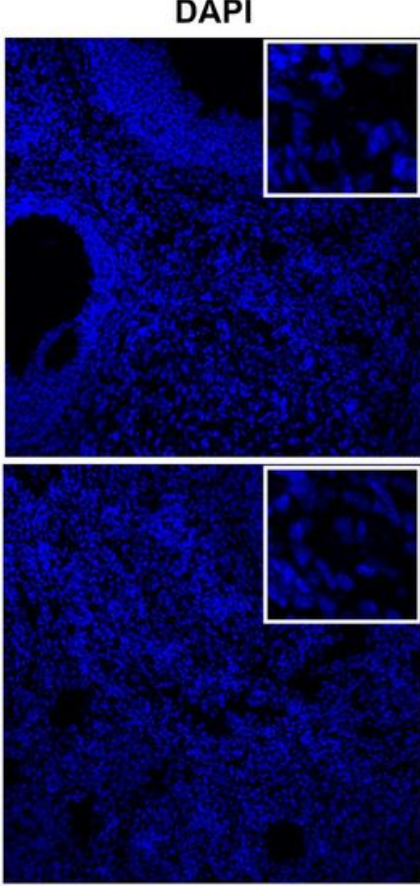

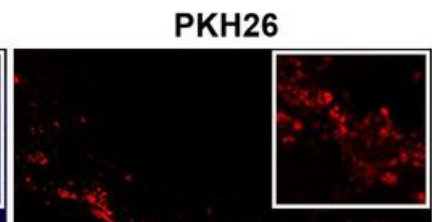

b

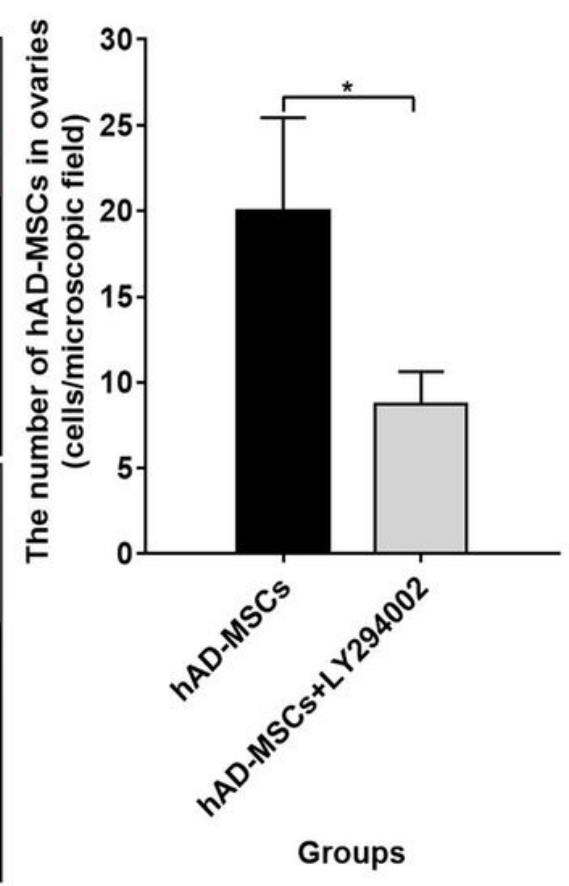

Figure 10

Inhibition of PI3K/Akt signaling pathway reduces hAD-MSC homing in vivo. a Transplanted PKH26labeled hAD-MSCs were observed in ovaries at $24 \mathrm{~h}$ after cell transplantation in the hAD-MSCs and hADMSCs+LY294002 groups by confocal microscopy analysis $(\times 200$ and $\times 800)$. b The number of hAD-MSCs was counted and compared in the hAD-MSCs and hAD-MSCs+LY294002 groups $(n=10)$. Representative images are shown. The yellow arrows indicate transplanted hAD-MSCs. ${ }^{*} \mathrm{P}<0.05$. Scale bars $=100 \mu \mathrm{m}$. 